\title{
Accretion disks around black holes in modified strong gravity
}

\author{
D. Pérez ${ }^{1}$, G. E. Romero ${ }^{1,2}$, and S. E. Perez Bergliaffa ${ }^{3}$
}

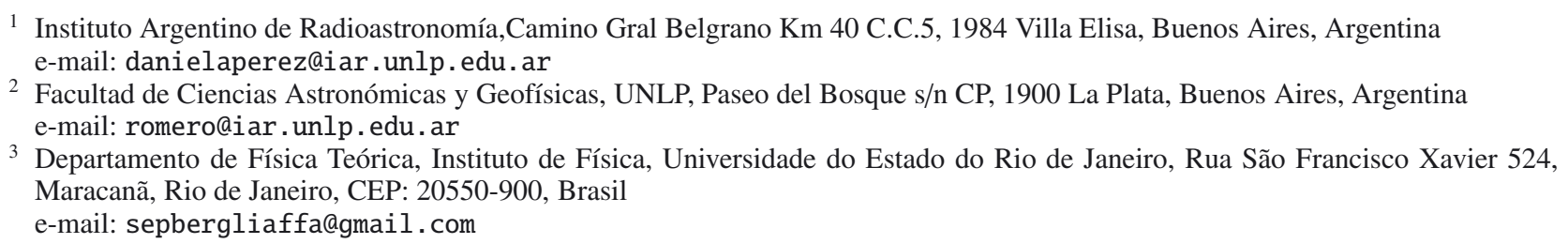

Received 13 September 2012 / Accepted 18 November 2012

\section{ABSTRACT}

\begin{abstract}
Context. Stellar-mass black holes offer what is perhaps the best scenario to test theories of gravity in the strong-field regime. In particular, $f(R)$ theories, which have been widely discus in a cosmological context, can be constrained through realistic astrophysical models of phenomena around black holes.

Aims. We aim at building radiative models of thin accretion disks for both Schwarzschild and Kerr black holes in $f(R)$ gravity. Methods. We study particle motion in $f(R)$-Schwarzschild and Kerr space-times.

Results. We present the spectral energy distribution of the accretion disk around constant Ricci scalar $f(R)$ black holes, and constrain specific $f(R)$ prescriptions using features of these systems.

Conclusions. A precise determination of both the spin and accretion rate onto black holes along with X-ray observations of their thermal spectrum might allow to identify deviations of gravity from general relativity. We use recent data on the high-mass X-ray binary Cygnus X-1 to restrict the values of the parameters of a class of $f(R)$ models.
\end{abstract}

Key words. black hole physics - accretion, accretion disks - gravitation

\section{Introduction}

General relativity (GR) is consistent, in some cases with good precision, with observational results (see for instance Will 2006). However, an energy momentum tensor representing exotic matter (loosely called "dark energy", e.g. Li et al. 2011) must be introduced in the right hand side of Einstein's equations to fit the currently available data when these are interpreted in the framework of the standard cosmological model (based on GR). Dark energy can be modelled by a cosmological constant, or by a scalar field with an equation of state given by $p=\omega_{\mathrm{DE}} \rho$, where $\omega_{\mathrm{DE}}<-1 / 3$ (Biswas et al. 2010a,b). None of these descriptions is free of problems, since the energy density associated with the cosmological constant that is inferred from astronomical observations is approximately 120 orders of magnitude lower than the value predicted by field theory (e.g. Weinberg 1989; Capozziello $\&$ Faraoni 2010), whereas the scalar field has features that are at odds with the scalar fields of particle physics (Sotiriou \& Faraoni 2010).

A different approach to explain the cosmological data is to modify the field equations of the gravitational field, in such a way that the ensuing theory differs from Einstein's in the lowcurvature regime. Since there is no a priori fundamental reason to restrict the gravitational Lagrangian to a linear function of the Ricci scalar $R$ (see for instance Magnano et al. 1987), more general theories can be formulated using nonlinear functions of this scalar. The so-called $f(R)$ theories (e.g. Capozziello \& Faraoni 2010) were first used to mimic the inferred accelerated expansion of the universe by Capoziello (2002). Currently there is in the literature a handful of $f(R)$-models in agreement with available data (De Felice \& Tsujikawa 2010).
Although the present revival of $f(R)$ theories is mainly due to their use in the description of phenomena that take place for low values of the Riemann curvature, these theories have also been applied to gravity in the opposite regime. As there is no direct evidence of the behaviour of the gravitational field for very large values of the curvature, the early universe and compact objects offer the possibility to find deviations from GR. Among the studies in modified gravity in the strong regime, we can mention the successful inflationary model based on the $R+\alpha R^{2}$ theory (Starobinsky 1980), and the related studies of reheating (Motohashi \& Nishizawa 2012) and particle production (Arbuzova et al. 2012) in the early universe. Also of importance is the treatment of neutron stars (Cooney et al. 2010) and black hole solutions.

Different aspects of black hole physics in $f(R)$ theories have been discussed in the literature by Psaltis et al. (2008a), Hendi \& Momeni (2011), Myung (2011), Myung et al. (2011), Moon et al. (2011a,b), Hendi et al. (2012), and Habib Mazharimousavi et al. (2012). Static and spherically symmetric black hole solutions were obtained via perturbation theory by de la Cruz-Dombriz et al. (2009), whereas black holes with these symmetries have been studied by means of a near-horizon analysis by Perez Bergliaffa \& De Oliveira (2011). Finally, $f(R)$-Kerr-Newman black holes solutions with constant Ricci scalar have been recently studied by Cembranos and collaborators (Cembranos et al. 2011).

From an astrophysical point of view, thin accretion for the Schwarzschild space-time in $f(R)$-gravity has been discussed by Pun et al. (2008), without including the expected spectra of concrete astrophysical black holes or any comparison with observational data. 
In the present work we investigate the existence of stable circular orbits in Schwarzschild and Kerr $f(R)$ space-times with constant Ricci scalar and analyze the main features of accretion disks around these black holes. In particular, we present temperature and spectral energy distributions for Page-Thorne disks, and compare the results with those obtained using the standard Shakura-Sunyaev model.

The paper is organized as follows. In Sect. 2 we provide a brief review of $f(R)$ theories of gravity. Circular orbits in both Schwarzschild and Kerr $f(R)$ space-times with constant Ricci scalar are studied in Sect. 3. Section 4 is devoted to the calculation of the properties of accretion disks in these space-times. Consequences for some specific prescriptions for the function $f$ are discussed in Sect. 5. We close with some considerations on the potential of astronomical observations to test the strong-field regime of gravity.

\section{2. $f(R)$ gravity}

In $f(R)$ gravity, the Lagrangian of the Hilbert-Einstein action, given by:

$S[g]=\frac{c^{3}}{16 \pi G} \int R \sqrt{-g} \mathrm{~d}^{4} x$,

is generalized to:

$S[g]=\frac{c^{3}}{16 \pi G} \int(R+f(R)) \sqrt{-g} \mathrm{~d}^{4} x$,

where $g$ is the determinant of the metric tensor, and $f(R)$ is an arbitrary function of the Ricci scalar. In the metric formalism the field equations are obtained varying Eq. (2) with respect to the metric:

$R_{\mu \nu}\left(1+f^{\prime}(R)\right)-\frac{1}{2} g_{\mu \nu}(R+f(R))$

$+\left(\nabla_{\mu} \nabla_{v}-g_{\mu v} \square\right) f^{\prime}(R)+\frac{16 \pi G}{c^{4}} T_{\mu \nu}=0$,

where $R_{\mu \nu}$ is the Ricci Tensor, $\square \equiv \nabla_{\beta} \nabla^{\beta}, f^{\prime}(R)=\mathrm{d} f(R) / \mathrm{d} R$, and the energy momentum tensor is defined by:

$T_{\mu \nu}=\frac{-2}{\sqrt{-g}} \frac{\delta\left(\sqrt{-g} \mathcal{L}_{\mathrm{m}}\right)}{\delta g^{\mu \nu}}$.

Here, $\mathcal{L}_{\mathrm{m}}$ stands for the matter Lagrangian.

Equations (3) are a system of fourth-order nonlinear equations for the metric tensor $g_{\mu \nu}$. An important difference between them and the Einstein field equations is that in $f(R)$ theories the Ricci scalar $R$ and the trace $T$ of the energy momentum tensor are differentially linked, as can be seen by taking the trace of Eq. (3), which yields:

$R\left(1+f^{\prime}(R)\right)-2(R+f(R))-3 \square f^{\prime}(R)+\frac{16 \pi G}{c^{4}} T=0$.

Hence, depending on the form of the function $f$, there may be solutions with traceless energy-momentum tensor and nonzero Ricci scalar. This is precisely the case of black hole space-times in the absence of a matter source.

Notice that in the case of constant Ricci scalar $R_{0}$ without matter sources, Eqs. (3) can be re-written as:

$R_{\mu \nu}=\Lambda g_{\mu \nu}$, where:

$\Lambda \equiv \frac{f\left(R_{0}\right)}{f^{\prime}\left(R_{0}\right)-1}$

and, by Eq.(5):

$R_{0}=\frac{2 f\left(R_{0}\right)}{f^{\prime}\left(R_{0}\right)-1}$.

Hence, in this case, any $f(R)$ theory is formally, but not physically, equivalent to GR with a cosmological constant given by Eq. $(6)^{1}$.

We shall investigate the existence of stable circular orbits in Schwarzschild and Kerr $f(R)$ space-times with constant Ricci scalar in the next section.

\section{Circular orbits around a black hole in $f(R)$}

\section{1. $f(R)$-Schwarzschild space-time}

The Schwarzschild space-time metric in $f(R)$ theories with constant Ricci scalar $R_{0}$ takes the form (Cembranos et al. 2011):

$\mathrm{d} s^{2}=-\left[\left(c^{2}-\frac{2 G M}{r}\right)-\frac{c^{2} R_{0}}{12} r^{2}\right]$
$\mathrm{d} t^{2}+\frac{\mathrm{d} r^{2}}{\left[\left(1-\frac{2 M G}{c^{2} r}\right)-\frac{R_{0}}{12} r^{2}\right]}+r^{2}\left(\mathrm{~d} \theta^{2}+\sin ^{2} \theta \mathrm{d} \phi^{2}\right)$,

where $R_{0}$, given by Eq. (7), can take, in principle, any real value. Since we are looking for space-time metrics that may represent astrophysical black holes, we shall select those values of $R_{0}$ that lead to acceptable solutions.

The radius $r_{0}$ of the horizon follows from the condition $g_{00}\left(r_{0}\right)=0$. From Eq. (8), the values of $r$ corresponding to the event horizon satisfy:

$c^{2} R_{0} r^{3}-12 c^{2} r+24 G M=0$.

In terms of the following adimensional quantities:

$$
\begin{aligned}
\mathrm{x} & \equiv \frac{r}{r_{\mathrm{g}}}, \\
\mathrm{R}_{0} & \equiv R_{0} r_{\mathrm{g}}^{2},
\end{aligned}
$$

where $r_{\mathrm{g}}=G M / c^{2}$, this equation takes the form:

$R_{0} x^{3}-12 x+24=0$.

We show in Fig. 1 the Ricci scalar as a function of the radial coordinate of the event horizon. We see that for $R_{0} \in(0,4 / 9)$ there is an inner black hole event horizon and an outer cosmological horizon, whereas for $\mathrm{R}_{0} \leq 0$ there is only one black hole event horizon. The event and cosmological horizons collapse for $R_{0}=4 / 9$ and for larger values of the Ricci scalar naked singularities occur and hence, no black holes are possi$b^{2}$. We shall, then, restrict ourselves to the study of trajectories for $R_{0} \in(-\infty, 4 / 9]$.

1 Accretion through thick disks onto Schwarzschild and Kerr black holes with a repulsive cosmological constant was studied by Rezzolla et al. (2003), and Slaný \& Stuchlík (2005), respectively. These studies do not analyze spectra or compare results with observational data.

2 The results obtained in our analysis of $f(R)$-Schwarzschild space-time with constant Ricci scalar are consistent with those given by Stuchlík \& Hledík (1999) in Schwarzschild-de Sitter and Schwarzschild-anti de Sitter space-times. 
D. Pérez et al.: Accretion disks onto black holes

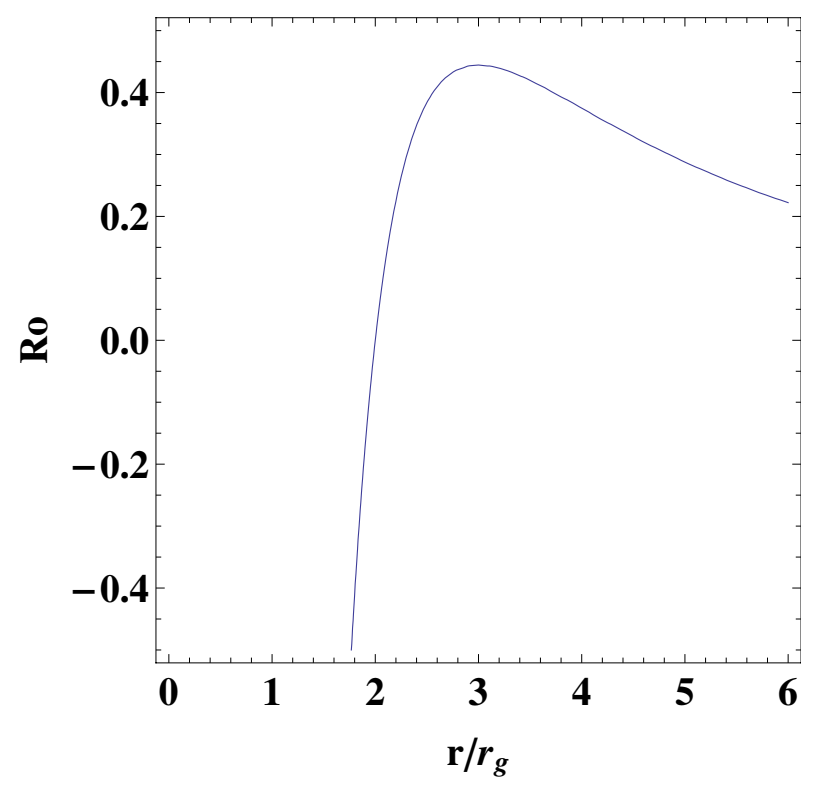

Fig. 1. Ricci scalar as a function of the radial coordinate of the event horizon in $f(R)$-Schwarzschild space-time.

\subsubsection{Equations of motion and effective potential in $f(R)$-Schwarzschild space-time}

The geodesic equations in the metric given in Eq. (8) can be obtained by means of the Euler-Lagrange equations using the Lagrangian:

$$
\begin{aligned}
L= & -\left[\left(c^{2}-\frac{2 G M}{r}\right)-\frac{c^{2} R_{0}}{12} r^{2}\right] \dot{t}^{2} \\
& +\frac{1}{\left[\left(1-\frac{2 G M}{c^{2} r}\right)-\frac{R_{0}}{12} r^{2}\right]} \dot{r}^{2}+r^{2}\left(\dot{\theta}^{2}+\sin \theta^{2} \dot{\phi}^{2}\right),
\end{aligned}
$$

where $\dot{x}^{\mu} \equiv \mathrm{d} x^{\mu} / \mathrm{d} \sigma$, and $\sigma$ is an affine parameter along the geodesic $x^{\mu}(\sigma)$. The resulting geodesic equations for $t$ and $\phi$ are (setting $\theta=\pi / 2$ ):

$$
\begin{aligned}
{\left[\left(1-\frac{2 G M}{c^{2} r}\right)-\frac{R_{0}}{12} r^{2}\right] \dot{t} } & =k, \\
r^{2} \dot{\phi} & =h,
\end{aligned}
$$

where $k$ and $h$ are constants. An equation for $r$ that is simpler than the one obtained from the Lagrangian follows from the modulus of the 4-momentum $\boldsymbol{p}$, given by $g_{\mu \nu} x^{\mu} x^{\nu}=\epsilon^{2}$, where $\epsilon^{2}=c^{2}$ for massive particles, and $\epsilon^{2}=0$ for photons. It takes the form:

$$
\begin{aligned}
-\left[\left(c^{2}-\frac{2 G M}{r}\right)-\frac{c^{2} R_{0}}{12} r^{2}\right] \dot{t}^{2} & \dot{r}^{2} \\
& +\frac{\left.\left.\frac{2 G M}{c^{2} r}\right)-\frac{R_{0}}{12} r^{2}\right]}{\left[\left(1-r^{2} \dot{\phi}^{2}=\epsilon^{2}\right.\right.},
\end{aligned}
$$

with $\dot{t}$ and $\dot{\phi}$ given by Eqs. (14) and (15), respectively. The set of Eqs. (14), (15), and (16) completely determine the motion of a particle in the $f(R)$-Schwarzschild space-time.

\subsubsection{Trajectories of massive particles}

Equations (14), (15), and (16) can be used to obtain the so-called energy equation (e.g. Hobson et al. 2006):

$$
\begin{aligned}
\dot{r}^{2}+\frac{h^{2}}{r^{2}}\left[\left(1-\frac{2 G M}{c^{2} r}\right)\right. & \left.-\frac{R_{0}}{12} r^{2}\right] \\
& +\left(-\frac{2 G M}{r}-\frac{c^{2} R_{0}}{12} r^{2}\right)=c^{2}\left(k^{2}-1\right) .
\end{aligned}
$$

The constant $k$ is defined as $k=E / m_{0} c^{2}$, where $E$ represents the total energy of the particle in its orbit, and $m_{0} c^{2}$ its rest mass energy. The constant $h$ stands for the angular momentum of the particle per unit mass. From Eq. (17) we can identify the effective potential per unit mass as:

$$
V_{\mathrm{eff}}(r)=\frac{h^{2}}{2 r^{2}}\left(1-\frac{2 G M}{c^{2} r}-\frac{R_{0}}{12} r^{2}\right)+\frac{1}{2}\left(-\frac{2 G M}{r}-\frac{c^{2} R_{0}}{12} r^{2}\right) .
$$

The extrema of the effective potential are obtained by looking for the roots of the derivative of the latter equation with respect to the radial coordinate. In terms of $\mathrm{x}, \mathrm{R}_{0}$, and the adimensional angular momentum per unit mass of the particle $\mathrm{h}=h\left(c r_{\mathrm{g}}\right)^{-1}$, this reads:

$\frac{\mathrm{d} V_{\text {eff }}}{\mathrm{dx}}=c^{2}\left(-\frac{\mathrm{h}^{2}}{\mathrm{x}^{3}}+\frac{3 \mathrm{~h}^{2}}{\mathrm{x}^{4}}+\frac{1}{\mathrm{x}^{2}}-\frac{\mathrm{R}_{0} \mathrm{x}}{12}\right)=0$.

The derivative of Eq. (19) with respect to $\mathrm{x}$ gives:

$\frac{\mathrm{d}^{2} V_{\mathrm{eff}}}{\mathrm{dx^{2 }}}=\frac{c^{2}}{\mu^{2}} \frac{\left(-4 \mathrm{R}_{0} \mathrm{x}^{4}+15 \mathrm{R}_{0} \mathrm{x}^{3}+12 \mathrm{x}-72\right)}{\mathrm{x}^{4}\left(-\frac{3}{\mathrm{x}}+1\right)}$,

where we have replaced $h$ by (Harko et al. 2009):

$\mathrm{h}=\mathrm{x}_{\mathrm{c}}{ }^{2} \frac{\sqrt{\frac{1}{\mathrm{x}_{\mathrm{c}}{ }^{3}}-\frac{\mathrm{R}_{0}}{12}}}{\sqrt{1-\frac{3}{\mathrm{x}_{\mathrm{c}}}}}$,

where $x_{c}$ corresponds to the radius of a circular orbit. We have performed the numerical calculation of the extrema of the effective potential for different values of $R_{0}$.

For $\mathrm{R}_{0}>0$, as shown by Stuchlík et al. (1999) and Rezzolla et al. (2003) in Schwarzschild-de Sitter space-time, stable circular orbits exist for values of the specific angular momentum that satisfy:

$h_{\text {isco }}<h<h_{\mathrm{osco}}$

where $h_{\text {isco }}$ and $h_{\text {osco }}$ stand for the local minimum and local maximum of the specific angular momentum at the inner and outer marginally stable radii. From Eq. (20) we see that the existence and location of the circular orbits depend on the Ricci scalar $R_{0}$. By equating Eq. (19) to zero and isolating $R_{0}$, we obtain the Ricci scalar as a function of the radial coordinate of the circular orbits:

$R_{0}=\frac{12\left(6-x_{c}\right)}{\left(15-4 x_{c}\right) x_{c}{ }^{3}}$

In Fig. 2 we show the plot of the latter equation. We see that there is an upper limit for the Ricci scalar, $R_{0}=2.85 \times 10^{-3}$, for which circular orbits are possible. In Fig. 3 we plot the effective potential as a function of the radial coordinate. The dots indicate 
Table 1. Location of the event and cosmological horizon, and of the innermost and outermost stable circular orbits for $\mathrm{R}_{0}>0$ in $f(R)$ Schwarzschild space-time.

\begin{tabular}{lcccc}
\hline \hline $\mathrm{R}_{0}$ & $\begin{array}{c}\text { Radius } \\
\text { event horizon }\end{array}$ & $\begin{array}{c}\text { Radius } \\
\text { cosmological horizon }\end{array}$ & $\begin{array}{c}\text { Radius innermost } \\
\text { stable circular orbit }\end{array}$ & $\begin{array}{c}\text { Radius outermost } \\
\text { stable circular orbit }\end{array}$ \\
\hline $10^{-12}$ & $\mathrm{x}_{\mathrm{eh}}=2$ & $\mathrm{x}_{\mathrm{ch}}=3.46 \times 10^{6}$ & $\mathrm{x}_{\text {isco }}=6$ & $\mathrm{x}_{\text {osco }}=14421.70$ \\
$10^{-6}$ & $\mathrm{x}_{\mathrm{eh}}=2$ & $\mathrm{x}_{\mathrm{ch}}=3463.10$ & $\mathrm{x}_{\text {isco }}=6.00016$ & $\mathrm{x}_{\text {osco }}=143.45$ \\
$10^{-4}$ & $\mathrm{x}_{\mathrm{eh}}=2$ & $\mathrm{x}_{\text {ch }}=345.40$ & $\mathrm{x}_{\text {isco }}=6.02$ & $\mathrm{x}_{\text {osco }}=30.16$ \\
$10^{-3}$ & $\mathrm{x}_{\mathrm{eh}}=2.00067$ & $\mathrm{x}_{\text {ch }}=108.53$ & $\mathrm{x}_{\text {isco }}=6.19$ & $\mathrm{x}_{\text {osco }}=13.17$ \\
$2 \times 10^{-3}$ & $\mathrm{x}_{\mathrm{eh}}=2.00134$ & $\mathrm{x}_{\mathrm{ch}}=76.44$ & $\mathrm{x}_{\text {isco }}=6.51$ & $\mathrm{x}_{\text {osco }}=9.80$ \\
$2.84 \times 10^{-3}$ & $\mathrm{x}_{\mathrm{eh}}=2.0019$ & $\mathrm{x}_{\mathrm{ch}}=63.98$ & $\mathrm{x}_{\text {isco }}=7.40$ & $\mathrm{x}_{\text {osco }}=7.60$ \\
\hline
\end{tabular}

Notes. Here $\mathrm{x} \equiv r / r_{\mathrm{g}}$.

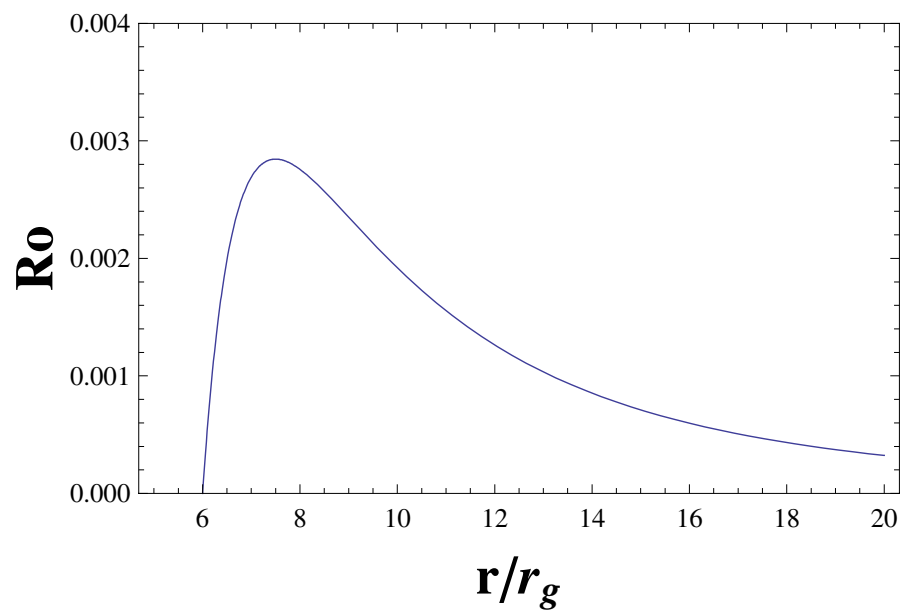

Fig. 2. Function given by Eq. (23). The absolute maximum corresponds to $\mathrm{x}=15 / 2$ and $\mathrm{R}_{0}=2.85 \times 10^{-3}$.

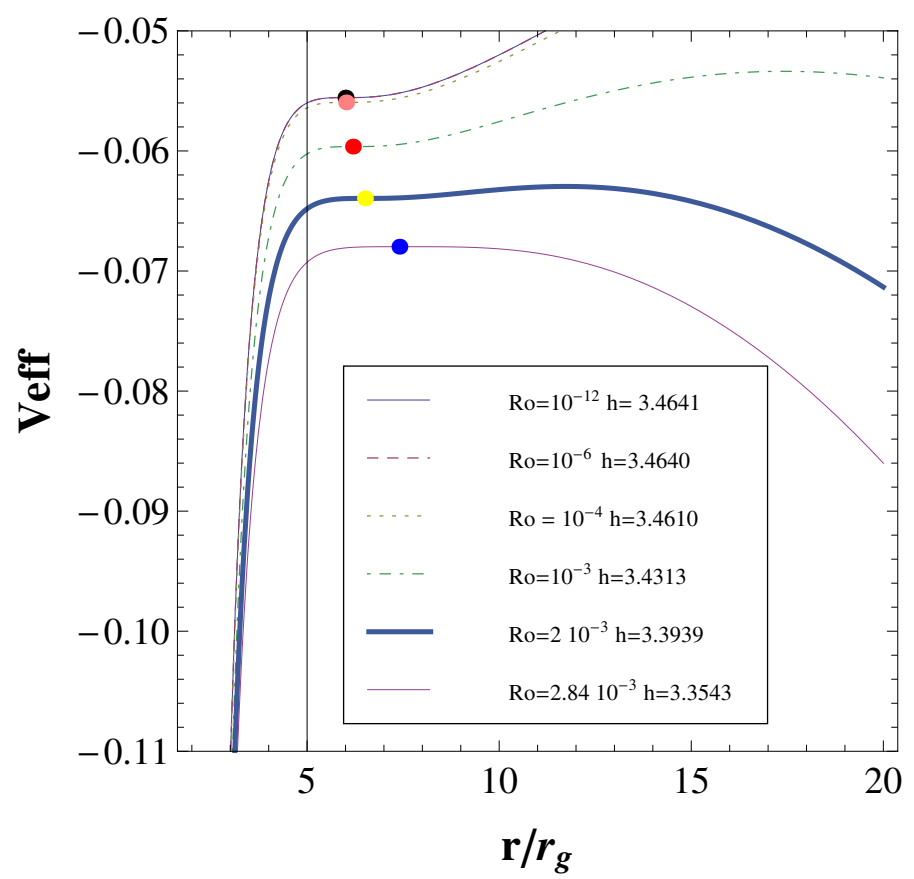

Fig. 3. Effective potential for different values of $\mathrm{R}_{0}>0$ and $\mathrm{h}$ in $f(R)$ Schwarzschild space-time. The dots indicate the location of the innermost stable circular orbit.

the location of the innermost stable circular orbits. The corresponding values of the event and cosmological horizons, radii of

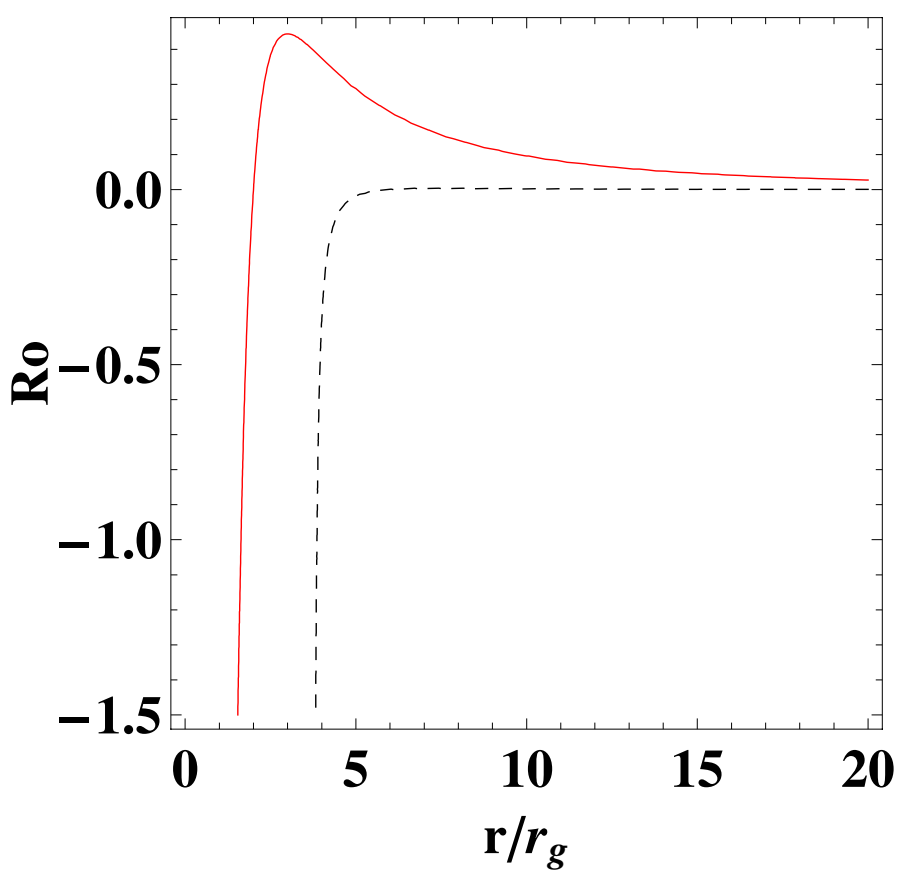

Fig. 4. Ricci scalar as a function of the radial coordinate of the event horizon (line) and of the Ricci scalar as a function of the radial coordinate of the innermost stable circular orbits (dashed line) for $\mathrm{R}_{0} \in$ $[-1.5,0.45]$ in $f(R)$-Schwarzschild space-time.

the innermost and outermost stable circular orbits, for six different values of $R_{0} \in\left(0,2.85 \times 10^{-3}\right)$ are shown in Table 1 . We see that for increasing values of the Ricci scalar the event horizon becomes larger than in Schwarzschild space-time in GR as well as the location of the innermost stable circular orbit.

The extrema of the effective potential for $\mathrm{R}_{0}<0$ are all located outside the event horizon, as shown in Fig. 4. The value of the radial coordinate for the event horizon is less than 2 (i.e. smaller than for Schwarzschild black holes in GR). The location of the innermost stable circular orbit is closer to the horizon than that of the Schwarzschild solution in Einstein's gravity. The limit $R_{0} \rightarrow-\infty$ in Eq. (20) yields:

$x^{3}(-4 x+15)=0 \Rightarrow x=0 \quad \vee \quad x=3.75$

The plot of the effective potential corresponding to the values of the parameters of Table 2 is shown in Fig. 5.

\subsection{Kerr space-time in $f(R)$ theories}

The axisymmetric, stationary and constant Ricci scalar geometry that describes a black hole with mass, electric charge and angular 
Table 2. Location of the event horizon, and of the innermost stable circular orbit for $\mathrm{R}_{0}<0$ in $f(R)$-Schwarzschild space-time.

\begin{tabular}{lcc}
\hline \hline $\mathrm{R}_{0}$ & $\begin{array}{c}\text { Radius } \\
\text { event horizon }\end{array}$ & $\begin{array}{c}\text { Radius innermost } \\
\text { stable circular orbit }\end{array}$ \\
\hline$-10^{-3}$ & $\mathrm{X}_{\mathrm{eh}}=1.999$ & $\mathrm{X}_{\text {isco }}=5.86$ \\
$-10^{-2}$ & $\mathrm{X}_{\mathrm{eh}}=1.993$ & $\mathrm{X}_{\text {isco }}=5.26$ \\
$-10^{-1}$ & $\mathrm{X}_{\mathrm{eh}}=1.939$ & $\mathrm{X}_{\text {isco }}=4.35$ \\
-1.5 & $\mathrm{X}_{\mathrm{eh}}=1.541$ & $\mathrm{X}_{\text {isco }}=3.83$ \\
\hline
\end{tabular}

Notes. Here $\mathrm{x} \equiv r / r_{\mathrm{g}}$.

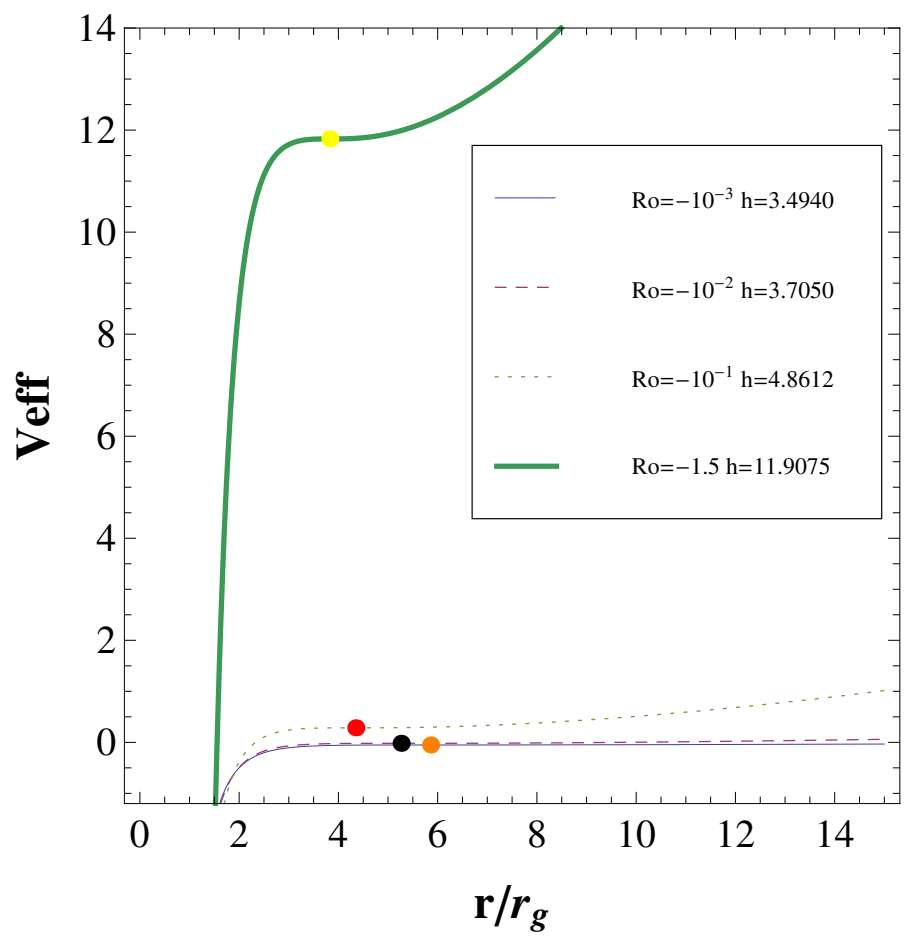

Fig. 5. Effective potential for different values of $\mathbf{R}_{0}<0$ and $\mathrm{h}$ in $f(R)$ Schwarzschild space-time. The dots indicate the location of the innermost stable circular orbit.

momentum was found by Carter (1973), and was used to study $f(R)$ black holes by Cembranos et al. (2011). The form of the metric is the following:

$$
\begin{aligned}
\mathrm{d} s^{2}= & \frac{\rho^{2}}{\Delta_{\mathrm{r}}} \mathrm{d} r^{2}+\frac{\rho^{2}}{\Delta_{\theta}} \mathrm{d} \theta^{2} \\
& +\frac{\Delta_{\theta} \sin ^{2} \theta}{\rho^{2}}\left[a \frac{c \mathrm{~d} t}{\Xi}-\left(r^{2}+a^{2}\right) \frac{\mathrm{d} \phi}{\Xi}\right]^{2} \\
& -\frac{\Delta_{\mathrm{r}}}{\rho^{2}}\left(\frac{c \mathrm{~d} t}{\Xi}-a \sin ^{2} \theta \frac{\mathrm{d} \phi}{\Xi}\right)^{2},
\end{aligned}
$$

where:

$$
\begin{aligned}
\Delta_{\mathrm{r}} & =\left(r^{2}+a^{2}\right)\left(1-\frac{R_{0}}{12} r^{2}\right)-\frac{2 G M r}{c^{2}}, \\
\rho^{2} & =r^{2}+a^{2} \cos \theta^{2} \\
\Delta_{\theta} & =1+\frac{R_{0}}{12} a^{2} \cos ^{2} \theta, \\
\Xi & =1+\frac{R_{0}}{12} a^{2} .
\end{aligned}
$$

Here $M$ and $a$ denote the mass and angular momentum per unit mass of the black hole, respectively, and $R_{0}$ is given by Eq.(7).

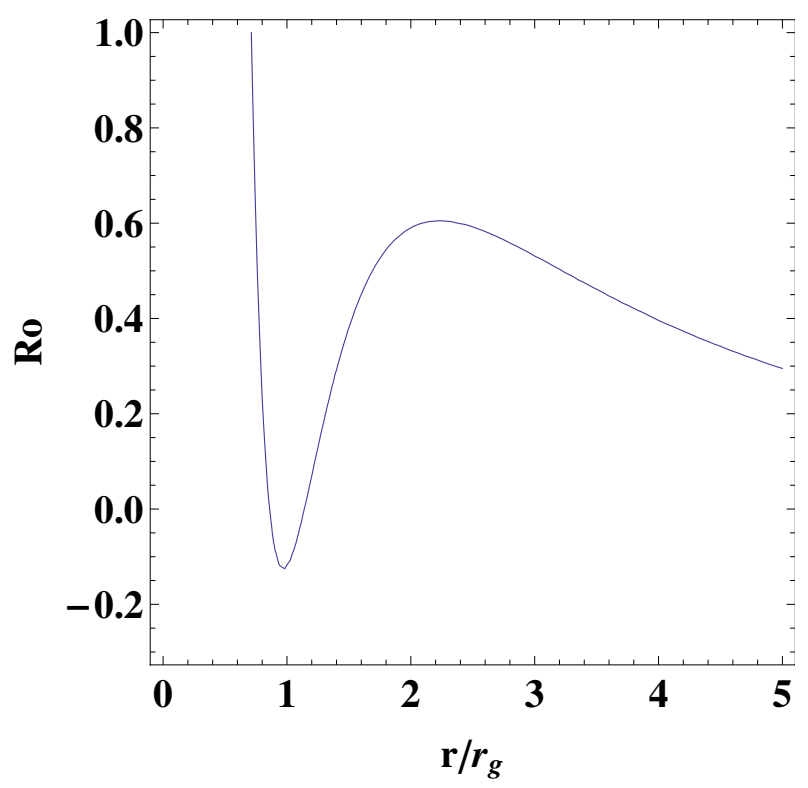

Fig. 6. Ricci scalar as a function of the radial coordinate of the event horizon for $\mathrm{R}_{0} \in[-0.3,1]$ and $\mathrm{a}=0.99$ in $f(R)$-Kerr space-time.

Because of the constancy of $p^{t}$ and $p^{\phi}$ along the trajectories, and of the reflection-symmetry of the metric through the equatorial plane, the orbit of any particle with initial condition $p^{\theta}=0$ will remain in the plane $\pi / 2$, where the metric has the form:

$$
\begin{aligned}
\mathrm{d} s^{2}= & -\frac{c^{2}}{r^{2} \Xi^{2}}\left(\Delta_{r}-a^{2}\right) \mathrm{d} t^{2}+\frac{r^{2}}{\Delta_{r}} \mathrm{~d} r^{2} \\
& -\frac{2 a c}{r^{2} \Xi^{2}}\left(r^{2}+a^{2}-\Delta_{r}\right) \mathrm{d} t \mathrm{~d} \phi \\
& +\frac{d \phi^{2}}{r^{2} \Xi^{2}}\left[\left(r^{2}+a^{2}\right)^{2}-\Delta_{r} a^{2}\right] .
\end{aligned}
$$

Here,

$\Delta_{r}=\left(r^{2}+a^{2}\right)\left(1-\frac{R_{0}}{12} r^{2}\right)-\frac{2 G M r}{c^{2}}$,

$$
\Xi=1+\frac{R_{0}}{12} a^{2} \text {. }
$$

If $R_{0} \rightarrow 0$, Eq. (30) represents the Kerr space-time metric in GR as expected.

The equation that yields the position of the event horizon is obtained by setting $1 / g_{\mathrm{rr}}=0$ :

$\Delta_{r}=\left(r^{2}+a^{2}\right)\left(1-\frac{R_{0}}{12} r^{2}\right)-\frac{2 G M r}{c^{2}}=0$.

In terms of $\mathrm{x}, \mathrm{R}_{0}$, and $\mathrm{a} \equiv a\left(r_{\mathrm{g}}\right)^{-1}$, this equation takes the form:

$\left(x^{2}+a^{2}\right)\left(1-\frac{R_{0} x^{2}}{12}\right)-2 x=0$.

In Fig. 6 we plotted the Ricci scalar as a function of the radial coordinate of the event horizon for $R_{0} \in[-0.3,1]$ and $a=0.99$ (i.e. a nearly maximally rotating black hole, such as Cygnus X1, Gou et al. 2011). If $R_{0} \in(0,0.6]$, there are 3 event horizons: the inner and outer horizons of the black hole and a cosmological horizon; for $\mathrm{R}_{0}>0.6$ there is a cosmological horizon that becomes smaller for larger values of $R_{0}$. If $R_{0} \in(-0.13,0)$ there are 2 event horizons. For $R_{0} \leq-0.13$ naked singularities occur. In the following we shall analyse the existence of stable circular orbits for $\mathrm{R}_{0} \in(-0.13,0.6]$. 


\subsubsection{Equations of motion and effective potential in $f(R)$-Kerr space-time}

In order to obtain an expression for the effective potential, we make use of the invariant length of the 4-momentum $p$ :

$g^{\mu v} p_{\mu} p_{v}=\epsilon^{2}$,

where $\epsilon^{2}=c^{2}$ for massive particles and $\epsilon^{2}=0$ for photons. Since we are only interested in trajectories on the equatorial plane, we set $p_{\theta}=0$, and Eq. (35) gives:

$g^{t t}\left(p_{t}\right)^{2}+2 g^{t \phi} p_{t} p_{\phi}+g^{\phi \phi} p_{\phi}^{2}+g^{r r} p_{r}^{2}=\epsilon^{2}$,

where:

$p_{t}=k c^{2}$,

$p_{\phi}=-h$,

$p_{r}=g_{r r} \dot{r}$.

Substituing Eqs. (37), (38), and (39) into (36), the equation for $\dot{r}^{2}$ takes the form:

$\dot{r}^{2}=g^{r r}\left[\epsilon^{2}-g^{t t}\left(k c^{2}\right)^{2}+2 g^{t \phi} h k c^{2}-g^{\phi \phi} h^{2}\right]$.

The contravariant components of the space-time metric given by Eq. (30) are:

$g^{t t}=\frac{\Xi^{2}}{\Delta_{r} c^{2} r^{2}}\left[\left(r^{2}+a^{2}\right)^{2}-\Delta_{r} a^{2}\right]$,

$g^{r r}=-\frac{\Delta_{r}}{r^{2}}$,

$g^{t \phi}=\frac{\Xi^{2}}{c \Delta_{r} r^{2}} a\left(r^{2}+a^{2}-\Delta_{r}\right)$,

$g^{\phi \phi}=-\frac{\Xi^{2}}{\Delta_{r} r^{2}}\left(\Delta_{r}-a^{2}\right)$.

Hence, the energy equation for a massive particle is given by:

$\frac{1}{2} \dot{r}^{2}+V_{\mathrm{eff}}\left(r, a, R_{0}, k, h\right)=\frac{c^{2}}{2}\left(k^{2}-1\right)$,

where the effective potential is:

$V_{\mathrm{eff}}\left(r, a, R_{0}, k, h\right)=\frac{c^{2} \Delta_{r}}{2 r^{2}}+\frac{c^{2}}{2}\left(k^{2}-1\right)-\frac{\Xi^{2}}{2 r^{4}} \Gamma$,

and:

$\Gamma \equiv\left[\left(r^{2}+a^{2}\right) c k-a h\right]^{2}-\Delta_{r}(a c k-h)^{2}$.

\subsubsection{Equatorial circular orbits of massive particles}

If a massive particle is moving in a circular orbit of radius $r_{\text {isco, }}$, the value of the effective potential at any point of the orbit satisfies the equation:

$V_{\mathrm{eff}}\left(r_{\mathrm{isco}}, a, R_{0}, k, h\right)=\frac{c^{2}}{2}\left(k^{2}-1\right)$,

so,

$\left.\frac{\mathrm{d} V_{\text {eff }}}{\mathrm{d} r}\left(r, a, R_{0}, k, h\right)\right|_{r_{\text {isco }}}=0$.

For a stable orbit, the equation:

$\left.\frac{\mathrm{d}^{2} V_{\mathrm{eff}}}{\mathrm{d} r^{2}}\left(r, a, R_{0}, k, h\right)\right|_{r_{\text {isco }}}>0$,
Table 3. Radii of event horizons and circular orbits for a $f(R)$-Kerr black hole of angular momentum $\mathrm{a}=0.99$, for some values of $\mathrm{R}_{0}<0$.

\begin{tabular}{lcc}
\hline \hline $\mathrm{R}_{0}$ & Radii event horizons & $\begin{array}{c}\text { Radius innermost } \\
\text { stable circular orbits }\end{array}$ \\
\hline$-10^{-3}$ & $\mathrm{x}_{\mathrm{eh} 1}=0.86, \mathrm{x}_{\mathrm{eh} 2}=1.14$ & $\mathrm{x}_{\mathrm{isco}}=1.452$ \\
$-1.2 \times 10^{-3}$ & $\mathrm{x}_{\mathrm{eh} 1}=0.86, \mathrm{x}_{\mathrm{eh} 2}=1.14$ & $\mathrm{x}_{\mathrm{isco}}=1.451$ \\
$-10^{-2}$ & $\mathrm{x}_{\mathrm{eh} 1}=0.86, \mathrm{x}_{\mathrm{eh} 2}=1.13$ & $\mathrm{x}_{\text {isco }}=1.43$ \\
$-10^{-1}$ & $\mathrm{x}_{\mathrm{eh} 1}=0.91, \mathrm{x}_{\mathrm{eh} 2}=1.03$ & $\mathrm{x}_{\text {isco }}=1.20$ \\
$-1.25 \times 10^{-1}$ & $\mathrm{x}_{\mathrm{eh} 1}=0.96, \mathrm{x}_{\mathrm{eh} 2}=0.98$ & $\mathrm{x}_{\text {isco }}=1.04$ \\
\hline
\end{tabular}

Notes. Here $\mathrm{X} \equiv r / r_{\mathrm{g}}$.

must also be satisfied. As shown in Sect. 3.2, there are black holes if $R_{0} \in(-0.13,0.6]$.

Numerical calculations of the radius of the innermost stable circular orbit for several values of $\mathrm{R}_{0}<0$, with $\mathrm{a}=0.99$, show that the stable circular orbits lay outside the event horizon (see Table 3). Notice that as the value of the scalar decreases, the radius of the innermost stable circular orbit becomes smaller. The radius of the innermost stable circular orbit in Kerr spacetime in GR ( $r_{\text {isco }}=1.4545 r_{\mathrm{g}}$, in the prograde case) is always larger than in $f(R)$-Kerr. In Fig. 7, the effective potential that correspond to the values of Table 3 are shown.

We follow Stuchlík \& Slaný (2004) to study the existence of stable circular orbits for $R_{0} \in(0,0.6]$. The specific angular momentum of a massive particle in a co-rotating circular orbit yields (Stuchlík \& Slaný 2004):

$h=-\frac{2 a+a x_{c}\left(x_{c}{ }^{2}+a^{2}\right) \frac{R_{0}}{12}-x_{c}\left(x_{c}{ }^{2}+a^{2}\right)\left(\frac{1}{x_{c}{ }^{3}}-\frac{R_{0}}{12}\right)^{1 / 2}}{x_{c}\left[1-\frac{3}{x_{c}}-\frac{a^{2} R_{0}}{12}+2 a\left(\frac{1}{x_{c}{ }^{3}}-\frac{R_{0}}{12}\right)^{1 / 2}\right]^{1 / 2}}$.

We see from the latter equation that circular orbits must satisfy the following two conditions:

$x_{c}<\left(\frac{12}{R_{0}}\right)^{1 / 3}$,

which is the same for $f(R)$-Schwarzschild space-time with positive Ricci scalar, and:

$1-\frac{3}{x_{c}}-\frac{a^{2} R_{0}}{12}+2 a\left(\frac{1}{x_{c}{ }^{3}}-\frac{R_{0}}{12}\right)^{1 / 2} \geq 0$.

The minimum and maximum of Eq. (51) give the values of the specific angular momentum that correspond to the innermost and outermost stable circular orbit respectively, once the angular momentum a of the black hole and $R_{0}$ are fixed. We show in Table 4 the values of such radii for different values of $R_{0}$, and $a=0.99$, and in Fig. 8 the corresponding plot of the effective potential. As expected, the radius of inner circular orbit is larger than in Kerr space-time in GR. We also found, by equating to zero the derivative of Eq. (51), that stable circular orbits only exist if $\mathrm{R}_{0} \in\left(0,1.45 \times 10^{-1}\right)$.

The analysis of the circular orbits presented in this section will be applied next to the construction of accretion disks around black holes.

\section{Accretion disks in strong gravity}

\subsection{Standard disk model in general relativity}

The first realistic model of accretion disks around black holes was formulated by Shakura \& Sunyaev (1973). They considered 


\section{Pérez et al.: Accretion disks onto black holes}

Table 4. Location of the event and cosmological horizon, and of the innermost and outermost stable circular orbits for $\mathrm{R}_{0}>0$ and $\mathrm{a}=0.99$ in $f(R)$-Kerr space-time.

\begin{tabular}{|c|c|c|c|c|}
\hline$\overline{\mathrm{R}_{0}}$ & $\begin{array}{c}\text { Radii } \\
\text { event horizons }\end{array}$ & $\begin{array}{c}\text { Radius } \\
\text { cosmological horizon }\end{array}$ & $\begin{array}{c}\text { Radius innermost } \\
\text { stable circular orbit }\end{array}$ & $\begin{array}{l}\text { Radius outermost } \\
\text { stable circular orbit }\end{array}$ \\
\hline $10^{-6}$ & $\mathrm{x}_{\mathrm{eh} 1}=0.86, \mathrm{x}_{\mathrm{eh} 2}=1.14$ & $x_{\mathrm{ch}}=3463$ & $\mathrm{x}_{\text {isco }}=1.4545$ & $\mathrm{x}_{\text {osco }}=143.59$ \\
\hline $10^{-4}$ & $\mathrm{x}_{\mathrm{eh} 1}=0.86, \mathrm{x}_{\mathrm{eh} 2}=1.14$ & $\mathrm{x}_{\mathrm{ch}}=345.40$ & $\mathrm{x}_{\mathrm{isco}}=1.4547$ & $\mathrm{x}_{\mathrm{osco}}=30.53$ \\
\hline $6.67 \times 10^{-4}$ & $\mathrm{x}_{\mathrm{eh} 1}=0.86, \mathrm{x}_{\mathrm{eh} 2}=1.14$ & $\mathrm{x}_{\mathrm{ch}}=113.12$ & $\mathrm{x}_{\mathrm{isco}}=1.4560$ & $x_{\text {osco }}=16.002$ \\
\hline $10^{-3}$ & $\mathrm{x}_{\mathrm{eh} 1}=0.86, \mathrm{x}_{\mathrm{eh} 2}=1.14$ & $x_{\mathrm{ch}}=108.53$ & $\mathrm{x}_{\mathrm{isco}}=1.4567$ & $\mathrm{x}_{\mathrm{osco}}=13.93$ \\
\hline $10^{-2}$ & $\mathrm{x}_{\mathrm{eh} 1}=0.85, \mathrm{x}_{\mathrm{eh} 2}=1.15$ & $x_{\mathrm{ch}}=33.59$ & $\mathrm{x}_{\mathrm{isco}}=1.4765$ & $\mathrm{x}_{\mathrm{osco}}=6.25$ \\
\hline $10^{-1}$ & $\mathrm{x}_{\mathrm{eh} 1}=0.83, \mathrm{x}_{\mathrm{eh} 2}=1.22$ & $x_{\mathrm{ch}}=9.80$ & $\mathrm{x}_{\text {isco }}=1.92$ & $x_{\text {osco }}=3.22$ \\
\hline
\end{tabular}

Notes. Here $\mathrm{x} \equiv r / r_{\mathrm{g}}$.

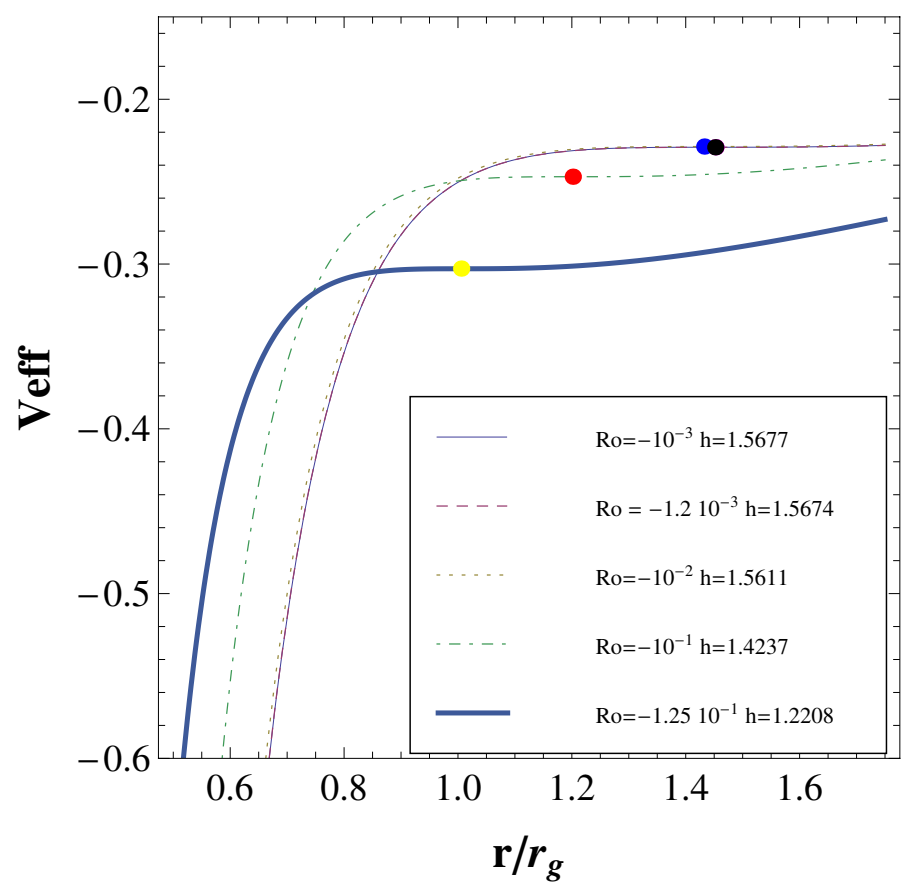

Fig. 7. Effective potential as a function of the radial coordinate $\left(\mathrm{R}_{0}<0\right.$, $\mathrm{a}=0.99)$, in $f(R)$-Kerr space-time. The dots indicate the location of the innermost stable circular orbit.

that the matter rotating in circular Keplerian orbits around the compact object loses angular momentum because of the friction between adjacent layers and spirals inwards. In the process gravitational energy is released, the kinetic energy of the plasma increases and the disk heats up, emitting thermal energy.

Novikov, Thorne, and Page (Novikov \& Thorne 1973; Page $\&$ Thorne 1974) made a relativistic analysis of the structure of an accretion disk around a black hole. They assumed the background space-time geometry to be stationary, axially-symmetric, asymptotically flat, and reflection-symmetric with respect to the equatorial plane. They also postulated that the central plane of the disk coincides with the equatorial plane of the black hole. This assumption entails that the metric coefficients $g_{t t}, g_{t \phi}, g_{r r}$, $g_{\theta \theta}$, and $g_{\phi \phi}$ depend only on the radial coordinate $r$.

The disk is supposed to be in a quasi-steady state (Novikov \& Thorne 1973), so any relevant quantity (for example the density or the temperature of the gas) is averaged over $2 \pi$, a proper radial distance of order $2 \mathrm{H}^{3}$, and the time interval $\Delta t$ that the gas takes

\footnotetext{
3 Here $H$ represents a particular height above the central plane of the $\operatorname{disk}(|z| \leq H \ll r)$.
}

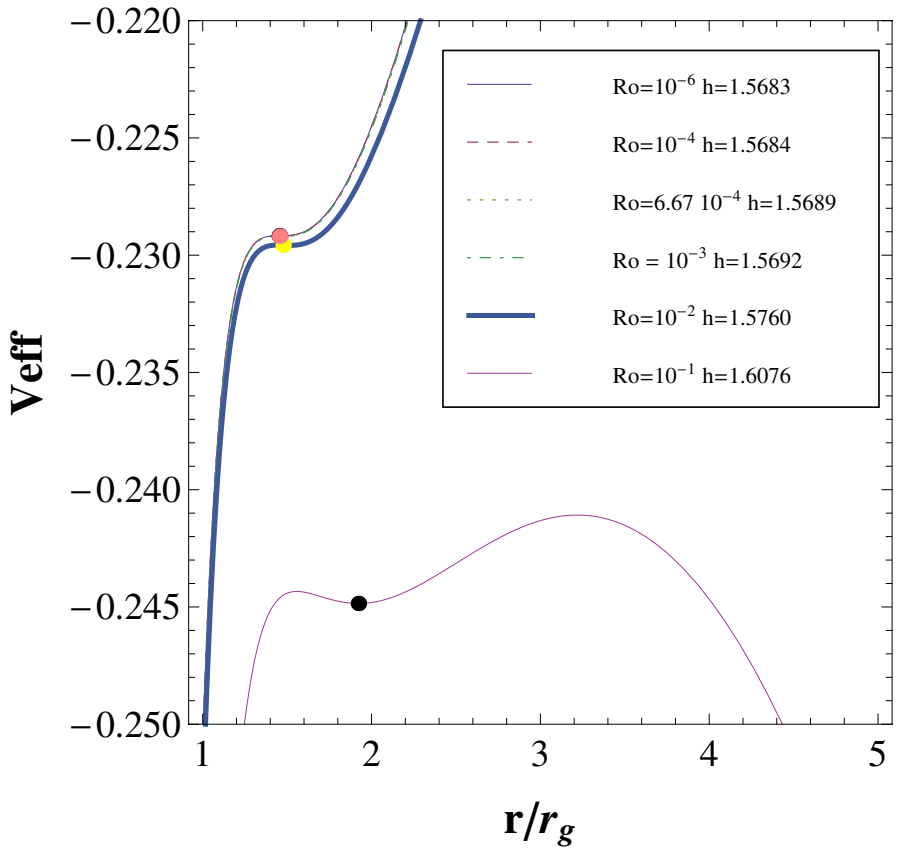

Fig. 8. Effective potential as a function of the radial coordinate $\left(R_{0}>0\right.$, $\mathrm{a}=0.99)$, in $f(R)$-Kerr space-time. The dots indicate the location of the innermost stable circular orbit.

to move inward a distance $2 H$. During $\Delta t$, the changes in the space-time geometry are negligible.

Particles move in the equatorial plane in nearly geodesic orbits; consequently, the gravitational forces exerted by the black hole completely dominate over the radial accelerations due to pressure gradients.

The expression of the energy flux for a relativistic accretion disk takes the form (Novikov \& Thorne 1973; Page \& Thorne 1974):

$Q(r)=-\frac{\dot{M}_{0}}{4 \pi \sqrt{-g}} \frac{\Omega, r}{(\widetilde{E}-\Omega \widetilde{L})^{2}} \int_{r_{\text {isco }}}^{r}(\widetilde{E}-\Omega \widetilde{L}) \widetilde{L, r} \mathrm{~d} r$

where $\dot{M}_{0}$ stands for the mass accretion rate, $\Omega$ for the angular velocity and $\widetilde{E}$ and $\widetilde{L}$ represent the specific energy and angular momentum, respectively. The lower limit of the integral $r_{\text {isco }}$ corresponds to the location of the innermost stable circular orbit.

The angular velocity $\Omega$, the specific energy $\widetilde{E}$, and the specific angular momentum $\widetilde{L}$ of the particles moving in circular 
orbits are given by (Harko et al. 2009):

$\Omega=\frac{\mathrm{d} \phi}{\mathrm{d} t}=\frac{-g_{t \phi, r}+\sqrt{\left(-g_{t \phi, r}\right)^{2}-g_{t t, r} g_{\phi \phi, r}}}{g_{\phi \phi, r}}$,

$\widetilde{E}=-\frac{g_{t t}+g_{t \phi} \Omega}{\sqrt{-g_{t t}-2 g_{t \phi} \Omega-g_{\phi \phi} \Omega^{2}}}$,

$\widetilde{L}=\frac{g_{t \phi}+g_{\phi \phi} \Omega}{\sqrt{-g_{t t}-2 g_{t \phi} \Omega-g_{\phi \phi} \Omega^{2}}}$.

Equations (56), and (57) can be derived by writing the effective potential $V_{\text {eff }}(r)$ in terms of the metric coefficients and solving for $\widetilde{E}$ and $\widetilde{L}$ the equations $V_{\mathrm{eff}}(r)=0$ and $V_{\mathrm{eff}, \mathrm{r}}(r)=0$. The formula for the angular velocity $\Omega=\mathrm{d} \phi / \mathrm{d} t$ is obtained by substituing $\widetilde{E}$, and $\widetilde{L}$ into the geodesic equations $\mathrm{d} t / \mathrm{d} \tau$ and $\mathrm{d} \phi / \mathrm{d} \tau$ (Harko et al. 2009).

In the next subsections we calculate the energy flux, temperature and luminosity of an accretion disk around a Schwarzschild and a Kerr black hole in GR and $f(R)$ gravity with constant Ricci scalar, adopting the following values for the relevant parameters: $M=14.8 M_{\odot}, \dot{M}=0.472 \times 10^{19} \mathrm{~g} \mathrm{~s}^{-1}$, and $\mathrm{a}=0.99$, which are the best estimates available for the wellknown galactic black hole Cygnus-X1 (Orosz et al. 2011; Gou et al. 2011).

\subsubsection{Relativistic accretion disk around Schwarzschild and Kerr black holes}

In order to obtain an expression of the energy flux and temperature of the disk, for the Schwarzschild black hole, we calculate the angular velocity $\Omega$, the specific energy $\widetilde{E}$ and angular momentum $\widetilde{L}$ of the particles in the disk, using the metric (Schwarzschild 1916):

$$
\begin{aligned}
\mathrm{d} s^{2}= & -c^{2}\left(1-\frac{2 G M}{c^{2} r}\right) \mathrm{d} t^{2}+\left(1-\frac{2 G M}{c^{2} r}\right)^{-1} \mathrm{~d} r^{2} \\
& +r^{2} \mathrm{~d} \theta^{2}+r^{2} \sin \theta^{2} \mathrm{~d} \phi^{2} .
\end{aligned}
$$

From Eqs. (55)-(57) we get:

$$
\begin{aligned}
& \Omega=\sqrt{\frac{G M}{r^{3}}}, \\
& \widetilde{E}=c \frac{\left(1-\frac{2 G M}{c^{2} r}\right)}{\sqrt{\left(1-\frac{3 G M}{c^{2} r}\right)}}, \\
& \widetilde{L}=\frac{\sqrt{G M} \sqrt{r}}{c \sqrt{\left(1-\frac{3 G M}{c^{2} r}\right)}} .
\end{aligned}
$$

By replacing these equations in Eq. (54), we obtain:

$$
\begin{aligned}
Q(\mathrm{x})= & \frac{3 \dot{M}_{0} c^{6}}{8 \pi \mathrm{x}^{7 / 2}} \frac{1}{(G M)^{2}}\left(1-\frac{3}{\mathrm{x}}\right)^{-1} \\
& \times\left[\sqrt{\mathrm{x}}+\sqrt{3} \tanh ^{-1} \sqrt{\frac{\mathrm{x}}{3}}\right]_{\mathrm{x} \text { isco }}^{\mathrm{x}},
\end{aligned}
$$

where $\mathrm{x}_{\text {isco }}=6 r_{\mathrm{g}}$ is the location of the innermost stable circular orbit in Schwarzschild space-time.

By means of Stefan-Bolzmann's law,

$$
T(r)=z\left(\frac{Q(r)}{\sigma_{\mathrm{SB}}}\right)^{1 / 4}
$$

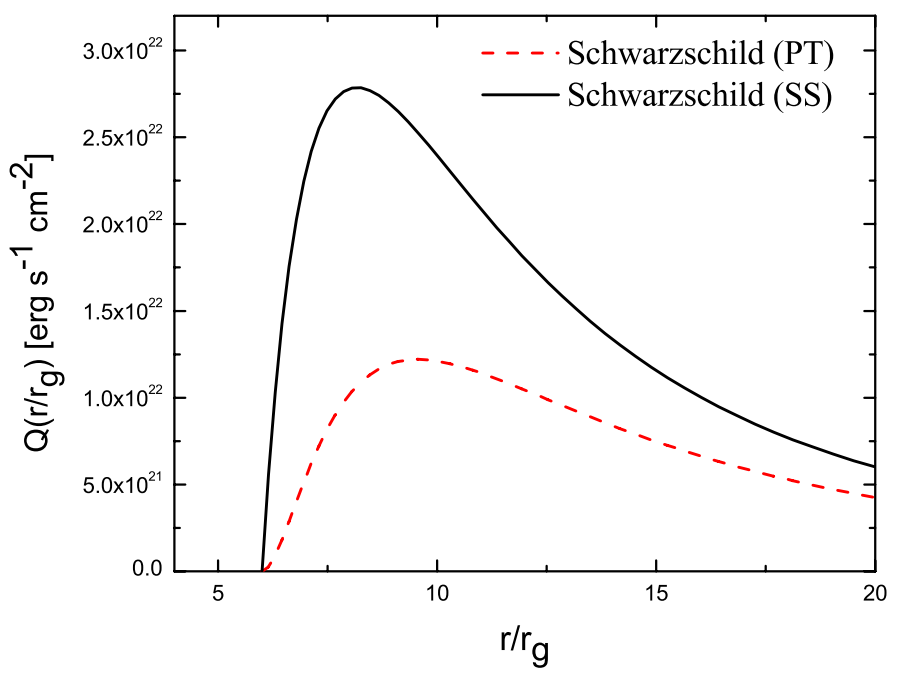

Fig. 9. Energy flux as a function of the radial coordinate of an accretion disk around a Schwarzschild black hole in Shakura-Sunyaev (SS) and Page-Thorne (PT) models, respectely.

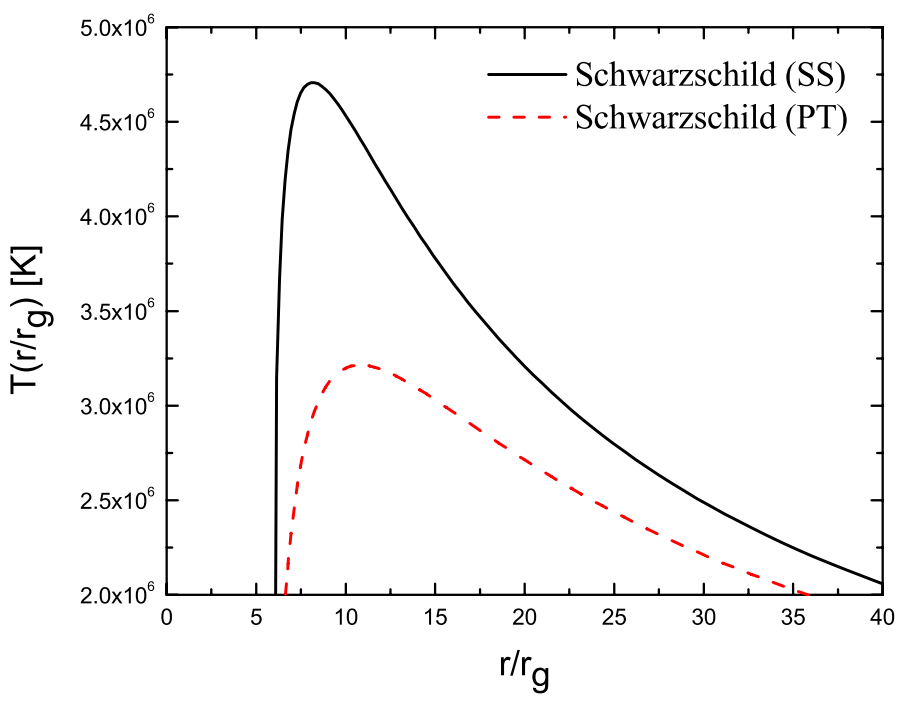

Fig. 10. Temperature as a function of the radial coordinate of an accretion disk around a Schwarzschild black hole in SS and PT models, respectively.

(where $z$ stands for the correction due to the gravitational redshift), the temperature of the disk can be obtained as a function of the radial coordinate $r$.

In Figs. 9 and 10 we plot the energy flux and the temperature as a function of the radial coordinate for a Schwarzschild black hole in both Keplerian and relativistic accretion disk models. We see that GR effects introduce a decrease of the peak of the energy flux by a factor $\approx 2$, and that the temperature distribution is also diminished.

The Kerr space-time metric (Kerr 1963) in Boyer-Lindquist coordinates for $\theta=\pi / 2$ is:

$$
\begin{aligned}
\mathrm{d} s^{2}= & -\frac{c^{2}}{r^{2}}\left(\Delta_{r}-a^{2}\right) d t^{2}+\frac{r^{2}}{\Delta_{r}} \mathrm{~d} r^{2} \\
& -\frac{2 a c}{r^{2}}\left(r^{2}+a^{2}-\Delta_{r}\right) \mathrm{d} t \mathrm{~d} \phi \\
& +\frac{d \phi^{2}}{r^{2}}\left[\left(r^{2}+a^{2}\right)^{2}-\Delta_{r} a^{2}\right],
\end{aligned}
$$


D. Pérez et al.: Accretion disks onto black holes

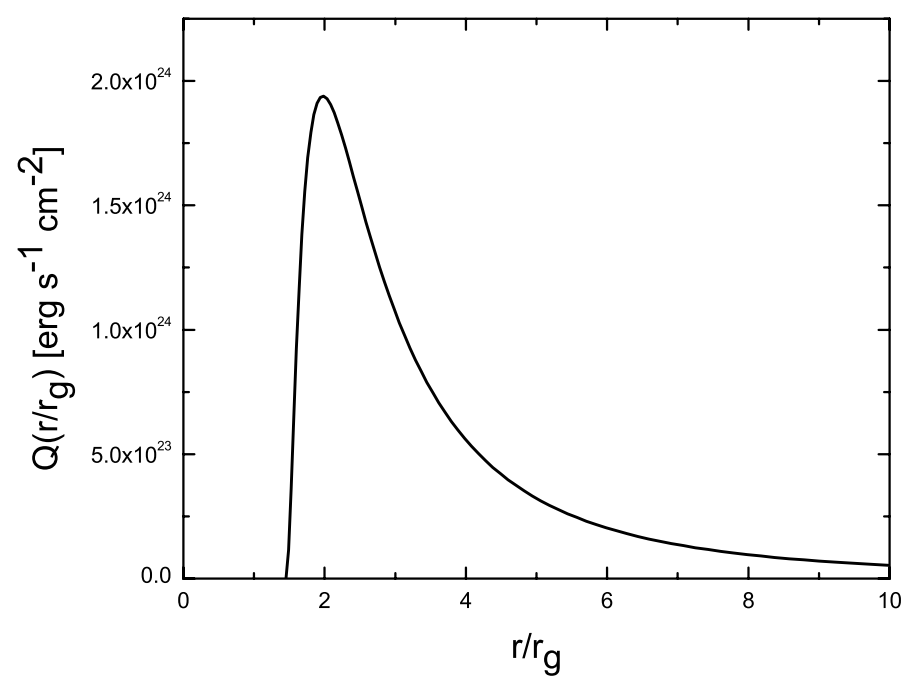

Fig. 11. Energy flux as function of the radial coordinate for an accretion disk around a Kerr black hole of angular momentum $a=0.99$ in the PT model.

where:

$\Delta_{r} \equiv\left(r^{2}+a^{2}\right)-\frac{2 G M r}{c^{2}}$.

The expression of the energy flux now becomes:

$Q(r)=-\frac{\dot{M}_{0}}{4 \pi \sqrt{-g}} \frac{\Omega, r}{(\widetilde{E}-\Omega \widetilde{L})^{2}} \int_{r_{\text {isco }}}^{r}(\widetilde{E}-\Omega \widetilde{L}) \widetilde{L_{, r}} \mathrm{~d} r$,

where:

$(\widetilde{E}-\Omega \widetilde{L}) \widetilde{L_{r}}=-\frac{c}{2} \frac{\left[\left(p_{1}+p_{2}\right) p_{3}\right]}{p_{4}}$,

$-\frac{\dot{M}_{0}}{4 \pi \sqrt{-g}} \frac{\Omega, r}{(\widetilde{E}-\Omega \widetilde{L})^{2}}=\frac{3 \dot{M}_{0}}{8 \pi} \frac{c^{2}}{\mu^{2}} \frac{p_{5}}{\left(p_{1}+p_{2}\right)^{2}}$,

and the coefficients $p_{i}$ are given by:

$p_{1}=x^{5 / 2}\left(1-\frac{2}{\mathrm{x}}+\frac{\mathrm{a}}{\mathrm{x}^{3 / 2}}\right)\left(-1+\frac{\mathrm{a}^{2}}{\mathrm{x}^{3}}\right)$,

$p_{2}=\left(-a+x^{3 / 2}\right)\left(1+\frac{a^{2}}{x^{2}}-2 \frac{a}{x^{3 / 2}}\right)$,

$p_{3}=\left(1+\frac{a}{x^{3 / 2}}\right)\left(-1+\frac{6}{x}-\frac{8 a}{x^{3 / 2}}+\frac{3 a^{2}}{x^{2}}\right)$,

$p_{4}=x^{3}\left(1-\frac{3}{x}+\frac{2 a}{x^{3 / 2}}\right)^{2}\left(-1+\frac{a^{2}}{x^{3}}\right)$,

$p_{5}=\frac{1}{\mathrm{x}^{3 / 2}}\left(\mathrm{x}^{3}+\mathrm{a}-2 \mathrm{ax} \mathrm{x}^{3 / 2}\right)\left(1-\frac{3}{\mathrm{x}}+\frac{2 \mathrm{a}}{\mathrm{x}^{3 / 2}}\right)$.

Here $\mathrm{x}=r / r_{\mathrm{g}}$ is an adimensional radial coordinate, and $\mathrm{a}=$ $a / r_{\mathrm{g}}$ is the angular momentum of the black hole in adimensional units.

In Figs. 11 and 12 we show the plots of the energy flux and temperature of an accretion disk around a Kerr black hole of angular momentum $\mathrm{a}=0.99$, whose innermost stable circular orbit is at $r_{\text {isco }}=1.4545 r_{\mathrm{g}}$. In Fig. 13 we show the luminosity of relativistic accretion disks around both Schwarzschild and Kerr black holes. For comparison, we also present the Schwarzschild/Shakura-Sunyaev luminosity.

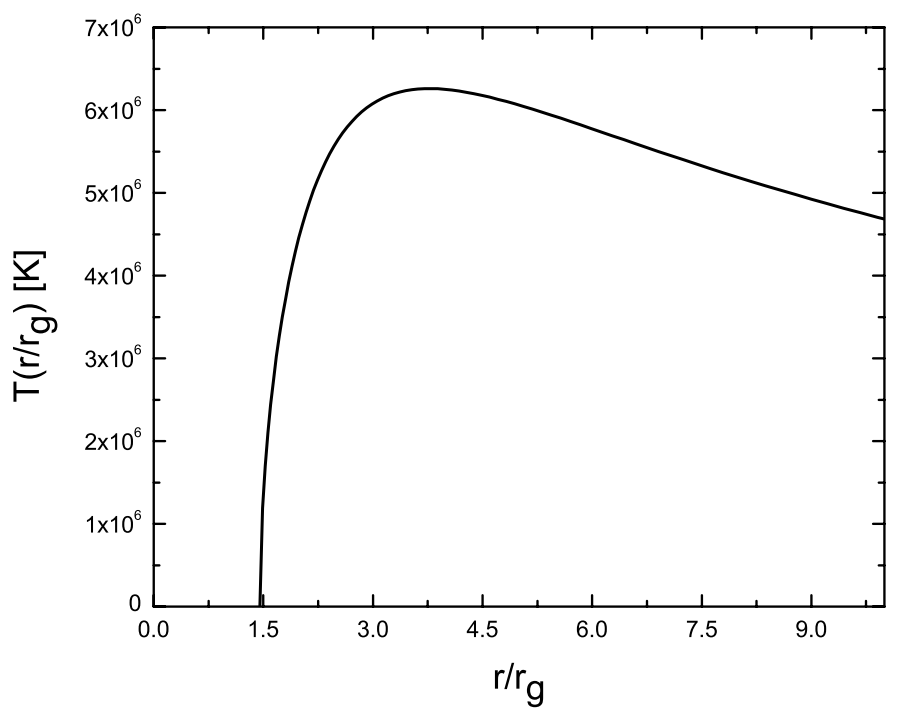

Fig. 12. Temperature as function of the radial coordinate of an accretion disk around a Kerr black hole of angular momentum $\mathrm{a}=0.99$ in the PT model.

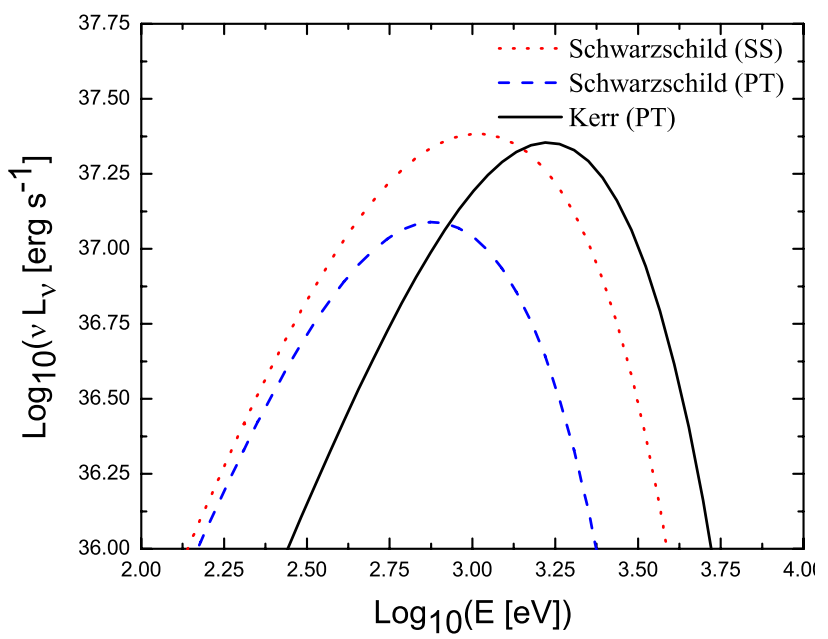

Fig. 13. Luminosity as function of the energy for a relativistic accretion disk around a Schwarzschild and a Kerr black hole $(a=0.99)$. We also plot the luminosity as a function of the energy of an accretion disk around a Schwarzschild black hole that corresponds to the SS model.

The values of the maximum temperature, luminosity, and the energy of the peak of the emission for all these models are shown in Table 5. As expected, the highest luminosity corresponds to a prograding accretion disk around a Kerr black hole. Since the last stable circular orbit is located closer to the black hole than in Schwarzschild space-time, thermal radiation is emitted at higher energies.

\section{2. $f(R)$-gravity}

\subsection{1. $f(R)$-Schwarzschild black holes}

The energy flux of an accretion disk around a $f(R)$ Schwarzschild black hole with metric given by Eq. (8) takes the form:

$Q=\frac{9 \dot{M}_{0} c^{6}}{4 \pi(G M)^{2}} \frac{\left(1-\frac{3}{\mathrm{x}}\right)^{-1}}{\mathrm{x}^{5} \sqrt{\frac{36}{\mathrm{x}^{3}}-3 c^{2} \mathrm{R}_{0}}} \int_{\mathrm{x}_{\mathrm{isco}}}^{\mathrm{x}}(\widetilde{E}-\Omega \widetilde{L}) \widetilde{L}_{\mathrm{r}} \mathrm{d} \mathrm{x}$, 
Table 5. Values of the energy of the peak of the emission, the maximum temperature, and luminosity of an accretion disk around Schwarzschild and Kerr black holes $(\mathrm{a}=0.99)$ in the SS and PT models.

\begin{tabular}{lccc}
\hline \hline & Schwarzschild (SS) & Schwarzschild (PT) & Kerr (PT) \\
\hline$E_{\max }$ & $1007.59 \mathrm{eV}$ & $746.94 \mathrm{eV}$ & $1654.9 \mathrm{eV}$ \\
$T_{\max }$ & $0.406 \mathrm{keV}$ & $0.277 \mathrm{keV}$ & $0.539 \mathrm{keV}$ \\
$L\left(E_{\max }\right)$ & $2.42 \times 10^{37} \mathrm{erg} \mathrm{s}^{-1}$ & $1.22 \times 10^{37} \mathrm{erg} \mathrm{s}^{-1}$ & $2.26 \times 10^{37} \mathrm{erg} \mathrm{s}^{-1}$ \\
\hline
\end{tabular}

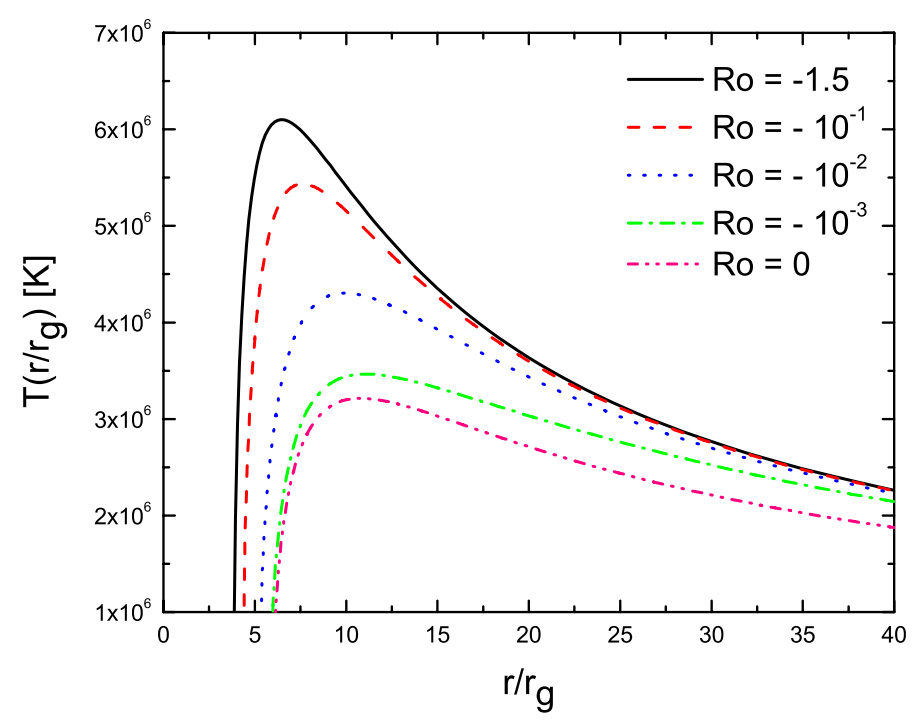

Fig. 14. Temperature as a function of the radial coordinate for some values of $\mathrm{R}_{0}<0$, for a $f(R)$-Schwarzschild black hole.

where

$(\widetilde{E}-\Omega \widetilde{L}) \widetilde{L}_{, \mathrm{r}}=-\frac{\sqrt{3}}{12 \mathrm{x}^{3}} \frac{\left(-12 \mathrm{x}+72+4 \mathrm{R}_{0} \mathrm{x}^{4}-15 \mathrm{R}_{0} \mathrm{x}^{3}\right)}{\left(1-\frac{3}{\mathrm{x}}\right)\left(\frac{12}{\mathrm{x}^{3}}-\mathrm{R}_{0}\right)^{1 / 2}}$.

We adopt for the radius of the outer edge of the disk (Dove et al. 1997):

$r_{\text {out }}=11 r_{\text {isco. }}$

According to the latter equation, if we take for the innermost stable circular orbit $r_{\text {isco }}=6.3 r_{\mathrm{g}}$, the outer egde of the disk yields approximately $70 r_{\mathrm{g}}$. If larger values of $r_{\text {out }}$ are considered, there are no major differences in the temperature and luminosity distributions.

We first compute the temperature and luminosity spectra distributions for $R_{0}<0$, and adopt the values given in Table 2 . In Figs. 14 and 15 we show the plots of the temperature as a function of the radial coordinate, and of the luminosity as a function of the energy, respectively. Notice that the corrections due to the gravitational redshift have been taken into account.

The maximum temperature as well as the luminosity increase for smaller values of $R_{0}$ (Figs. 14 and 15). In the four cases presented, the accretion disk is hotter than in GR, e.g. in a $f(R)$ Schwarzschild disk for $\mathrm{R}_{0}=-1.5$, the maximum temperature and luminosity are a factor 1.9 and 3.7, respectively, higher. The energy corresponding to the peak of the emission shifts to higher values, reaching $1359.20 \mathrm{eV}$ for the set of adopted parameters.

We showed in Sect. 3.1.2 that for $R_{0}>0$, stable circular orbits are possible within a minimum and maximum radius. We see in Table 1 that only for $R_{0}=10^{-12}$ and $R_{0}=10^{-6}$ accretion disks are possible, if we take for the radius of the outer edge of the disk $r_{\text {out }}=70 r_{\mathrm{g}}$. The values of the location of the

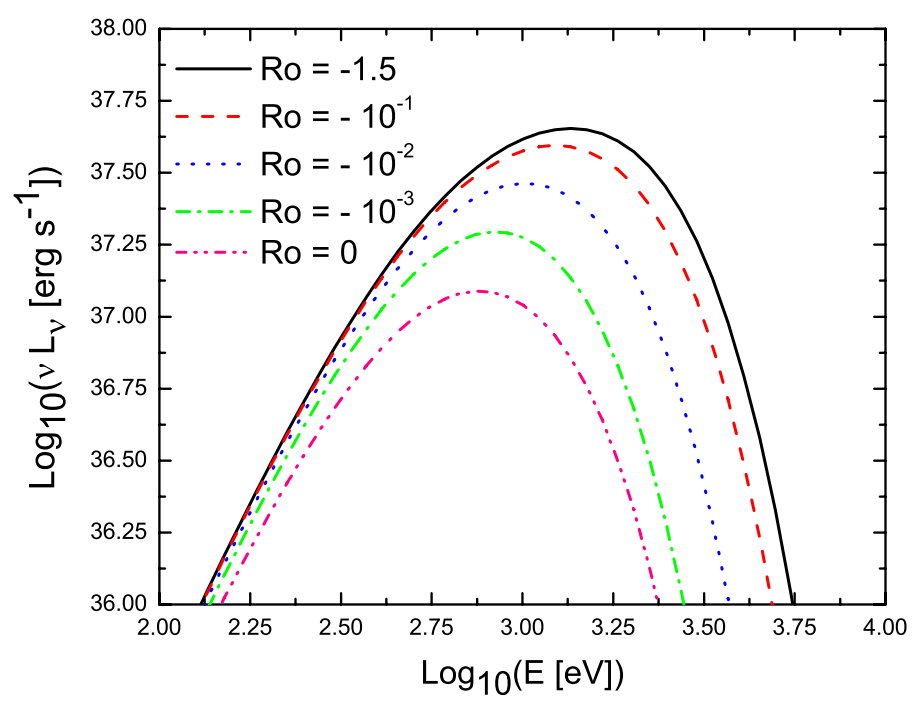

Fig. 15. Luminosity as a function of the energy for some values of $\mathrm{R}_{0}<$ 0 , for a $f(R)$-Schwarzschild black hole.

innermost stable circular orbit, location in the radial coordinate of the maximum temperature, maximum temperature and luminosity, and the energy of the peak of the emission are displayed in Table 7 . We conclude that for $R_{0} \in\left(0,10^{-6}\right.$ ] the temperature and energy distributions have no significant differences with Schwarzschild's distributions in GR.

\subsection{2. $f(R)$-Kerr black holes}

We calculate next the energy flux of an accretion disk around a $f(R)$-Kerr black hole:

$Q(\mathrm{x})=-\frac{\dot{M}_{0}}{4 \pi \sqrt{-g}} \frac{\Omega, \mathrm{x}}{(\widetilde{E}-\Omega \widetilde{L})^{2}} \int_{\mathrm{x}_{\mathrm{isco}}}^{\mathrm{x}}(\widetilde{E}-\Omega \widetilde{L}) \widetilde{L}_{\mathrm{,}} \mathrm{dx}$,

where:

$\Omega_{, \mathrm{x}}=-36 \sqrt{3} \frac{c}{\mu^{2}} \eta$,

$\eta \equiv \frac{\mathrm{x}^{1 / 2}\left\{12 \mathrm{x}^{3}+\mathrm{a}\left[12 \mathrm{a}-\mathrm{aR}_{0} \mathrm{x}^{3}-4 \sqrt{36 \mathrm{x}^{3}-3 \mathrm{x}^{6} \mathrm{R}_{0}}\right]\right\}}{\left(12 \mathrm{x}^{3}+\mathrm{a}^{2} \mathrm{R}_{0} \mathrm{x}^{3}-12 \mathrm{a}^{2}\right)^{2} \sqrt{-\mathrm{R}_{0} \mathrm{x}^{3}+12}}$,

$\widetilde{L}=\frac{2 \mu\left(l_{1}+l_{2}\right)}{\left(12+\mathrm{a}^{2} \mathrm{R}_{0}\right) \times \sqrt{l_{3}+l_{4}}}$

$\widetilde{L}_{, \mathrm{x}}=-\frac{4 \mathrm{x}\left[12 \mathrm{x}^{3}+\mathrm{a}^{2}\left(-12+\mathrm{R}_{0} \mathrm{x}^{3}\right)\right]\left(l_{5}+l_{6}+l_{7}+l_{8}+l_{9}\right)}{\left(12+\mathrm{a}^{2} \mathrm{R}_{0}\right) \sqrt{12 \mathrm{x}^{3}-\mathrm{R}_{0} \mathrm{x}^{6}}\left(l_{10}+l_{11}\right)^{3 / 2}}$,

$(\widetilde{E}-\Omega \widetilde{L})=\frac{12 c \sqrt{l_{3}+l_{4}}}{\left(12+\mathrm{a}^{2} \mathrm{R}_{0}\right)\left[12 \mathrm{x}^{3}+\mathrm{a}^{2}\left(-12+\mathrm{x}^{3} \mathrm{R}_{0}\right)\right]}$, 
D. Pérez et al.: Accretion disks onto black holes

Table 6. Values of the location of the last stable circular orbit, location in the radial coordinate of the maximum temperature, maximum temperature and luminosity, and the energy of the peak of the emission for an accretion disk around a $f(R)$-Schwarzschild black hole with $\mathrm{R}_{0}<0$.

\begin{tabular}{lccccc}
\hline \hline$f(R)-$ Schwarzschild & $\mathrm{R}_{0}=0$ & $\mathrm{R}_{0}=-10^{-3}$ & $\mathrm{R}_{0}=-10^{-2}$ & $\mathrm{R}_{0}=-10^{-1}$ & $\mathrm{R}_{0}=-1.5$ \\
\hline$r_{\text {isco }} / r_{\mathrm{g}}$ & 6 & 5.85 & 5.26 & 4.35 & 3.82 \\
$r_{\mathrm{Tmax}} / r_{\mathrm{g}}$ & 10.82 & 11.04 & 10.05 & 7.58 & 6.45 \\
$T_{\max }$ & $0.277 \mathrm{keV}$ & $0.298 \mathrm{keV}$ & $0.371 \mathrm{keV}$ & $0.468 \mathrm{keV}$ & $0.526 \mathrm{keV}$ \\
$E_{\max }$ & $746.94 \mathrm{eV}$ & $825.31 \mathrm{eV}$ & $1007.59 \mathrm{eV}$ & $1230.13 \mathrm{eV}$ & $1359.20 \mathrm{eV}$ \\
$L\left(E_{\max }\right)$ & $1.22 \times 10^{37} \mathrm{erg} \mathrm{s}^{-1}$ & $1.97 \times 10^{37} \mathrm{erg} \mathrm{s}^{-1}$ & $2.91 \times 10^{37} \mathrm{erg} \mathrm{s}^{-1}$ & $3.93 \times 10^{37} \mathrm{erg} \mathrm{s}^{-1}$ & $4.5 \times 10^{37} \mathrm{erg} \mathrm{s}^{-1}$ \\
\hline
\end{tabular}

Table 7. Values of the location of the innermost stable circular orbit, location in the radial coordinate of the maximum temperature, maximum temperature and luminosity, and the energy of the peak of the emission for an accretion disk around a $f(R)$-Schwarzschild black hole with $\mathrm{R}_{0}>0$.

\begin{tabular}{lcc}
\hline \hline$f(R)-$ Schwarzschild & $\mathrm{R}_{0}=0$ & $\mathrm{R}_{0}=10^{-6}$ \\
\hline$r_{\text {isco }} / r_{\mathrm{g}}$ & 6 & 6.00016 \\
$r_{\mathrm{T} \max } / r_{\mathrm{g}}$ & 10.81 & 10.82 \\
$T_{\max }$ & $0.277 \mathrm{keV}$ & $0.277 \mathrm{keV}$ \\
$E_{\max }$ & $746.94 \mathrm{eV}$ & $746.94 \mathrm{eV}$ \\
$L\left(E_{\max }\right)$ & $1.22 \times 10^{37} \mathrm{erg} \mathrm{s}^{-1}$ & $1.22 \times 10^{37} \mathrm{erg} \mathrm{s}^{-1}$ \\
\hline
\end{tabular}

and

$$
\begin{aligned}
& l_{1}=-72 \mathrm{a}^{3} \mathrm{x}-216 \mathrm{ax} \mathrm{x}^{3}+12 \mathrm{x}^{3} \sqrt{36 \mathrm{x}^{3}-3 \mathrm{x}^{6} \mathrm{R}_{0}}, \\
& l_{2}=a^{2} \sqrt{36 x^{3}-3 x^{6} R_{0}}\left[a^{2} x R_{0}+24+x\left(12+x^{2} R_{0}\right)\right], \\
& l_{3}=-432 a^{2} x^{2}+x^{6}\left(12+a^{2} R_{0}\right)^{2}+48 a^{3} \sqrt{36 x^{3}-3 x^{6} R_{0}}, \\
& l_{4}=144 a x^{2} \sqrt{36 x^{3}-3 x^{6} R_{0}} \\
& -12 x^{3}\left[36 x^{2}+a^{4} R_{0}+a^{2}\left(36-3 x^{2} R_{0}\right)\right], \\
& l_{5}=\left[108 \mathrm{ax}^{2}\left(-24-12 \mathrm{x}+5 \mathrm{R}_{0} \mathrm{x}^{3}\right)\right] \sqrt{12 \mathrm{x}^{3}-\mathrm{R}_{0} \mathrm{x}^{6}}, \\
& l_{6}=36 \mathrm{a}^{3}\left(-48-36 \mathrm{x}+7 \mathrm{R}_{0} \mathrm{x}^{3}\right) \sqrt{12 \mathrm{x}^{3}-\mathrm{R}_{0} \mathrm{x}^{6}}, \\
& l_{7}=\sqrt{3} a^{4} x\left(-12+R_{0} x^{3}\right)\left[-108+R_{0} x^{2}\left(-3+R_{0} x^{3}\right)\right] \text {, } \\
& l_{8}=36 \sqrt{3} \mathrm{x}^{5}\left\{72+\mathrm{x}\left[-12+\mathrm{R}_{0} \mathrm{x}^{2}(-15+4 \mathrm{x})\right]\right\}, \\
& l_{9}=3 \sqrt{3} a^{2} x^{2}\left\{864+x l_{9 a}\right\}, \\
& l_{9 a}=\left[2160+\mathrm{x} l_{9 a 1}\right], \\
& l_{9 a 1}=\left(432+\mathrm{R}_{0}^{2} \mathbf{x}^{4}(15+8 \mathrm{x})-12 \mathrm{R}_{0} \mathrm{x}(21+26 \mathrm{x})\right), \\
& l_{10}=144(-3+x) x^{5}+a^{4} R_{0} x^{3}\left(-12+R_{0} x^{3}\right) \\
& +48 a^{3} \sqrt{36 x^{3}-3 R_{0} x^{6}}, \\
& l_{11}=144 \mathrm{ax}^{2} \sqrt{36 \mathrm{x}^{3}-3 \mathrm{R}_{0} \mathrm{x}^{6}}+12 \mathrm{a}^{2} \mathrm{x}^{2} l_{11 a}, \\
& l_{11 a}=-36+\mathrm{x}\left[-36+\mathrm{R}_{0} \mathrm{x}^{2}(3+2 \mathrm{x})\right] \text {. }
\end{aligned}
$$

If we adopt for the radius of the inner edge of the disk $1.4545 r_{\mathrm{g}}$, according to Eq. (76), $r_{\text {out }} \approx 16 r_{\mathrm{g}}$. From Eq. (77) we numerically calculate the temperature and luminosity for the values shown in Table 3, taking into account the corrections coming from the gravitational redshift. The results are displayed in Figs. 16, 17, and Table 8. We see that the temperature of the disk increases for smaller values of $\mathrm{R}_{0}$. The ratio of the maximum temperature between the GR and $f(R)$ cases, with $\mathrm{R}_{0}=-1.25 \times 10^{-1}$, is 1.20 . The peak of the emission rises a factor of 2 , and the corresponding energy is shifted towards higher energies.

Since the radius of the outer edge of the disk is $16 r_{\mathrm{g}}$, we see from Table 4 that accretion disks are only possible for $R_{0}=10^{-6}$ and $R_{0}=10^{-4}$, until up $R_{0}=6.67 \times 10^{-4}$. For such values of $R_{0}$, we show in Table 9 the values of the location of the last stable circular orbit, maximum temperature, luminosity, and the energy

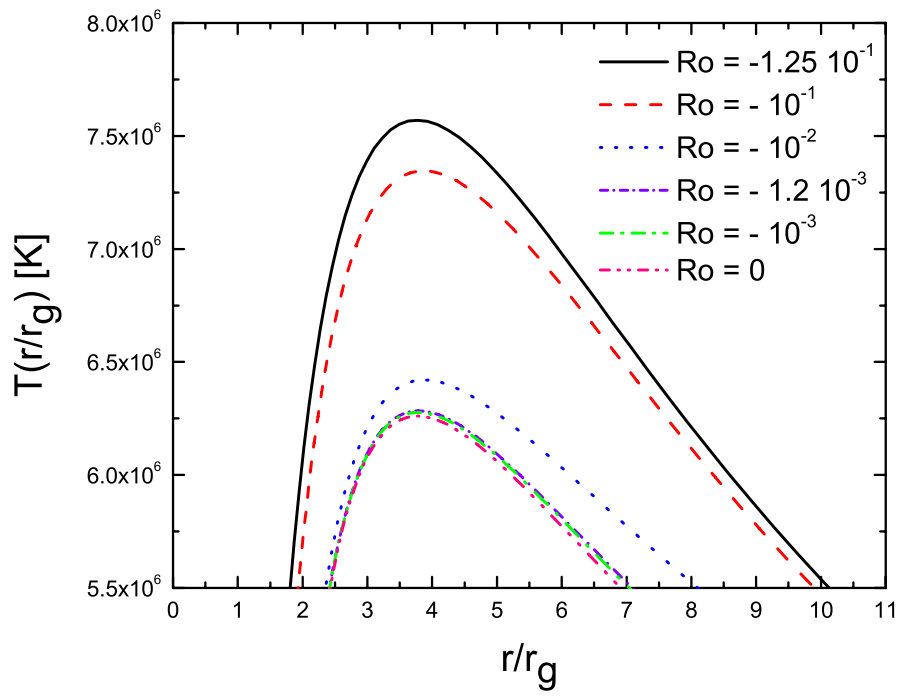

Fig. 16. Temperature as a function of the radial coordinate for some values of $\mathrm{R}_{0}<0$ of a $f(R)$-Kerr black hole of angular momentum $\mathrm{a}=$ 0.99 , corrected by gravitational redshift.

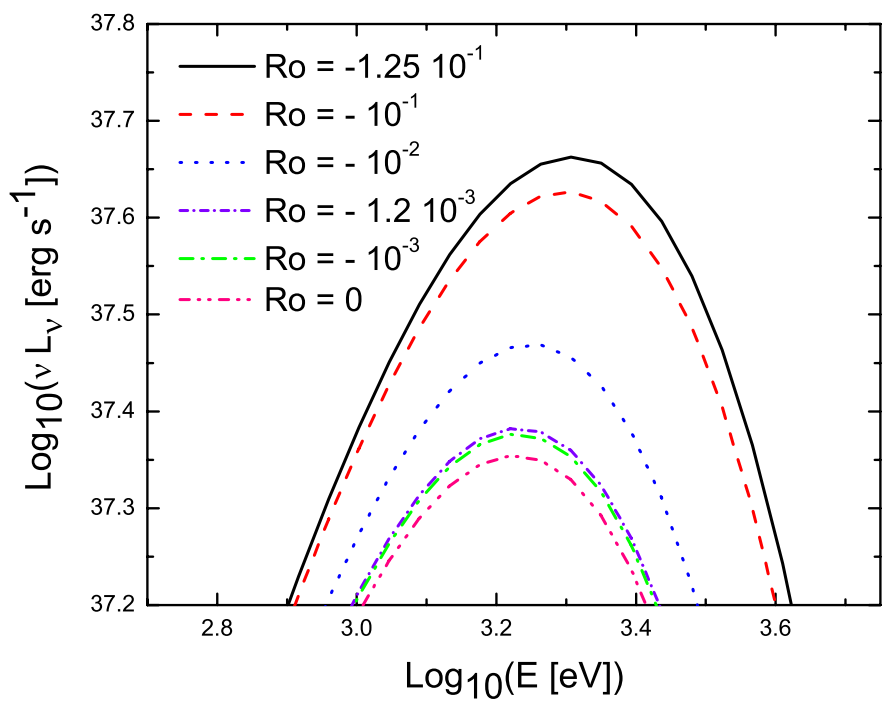

Fig. 17. Luminosity as a function of the energy for some values of $\mathrm{R}_{0}<$ 0 , for a $f(R)$-Kerr black hole of angular momentum $\mathbf{a}=0.99$. 
Table 8. Location of the last stable circular orbit and maximum temperature, maximum temperature, luminosity, and energy of the peak of the emission for an accretion disk around a $f(R)$-Kerr black hole with $\mathrm{R}_{0}<0$ and $\mathrm{a}=0.99$.

\begin{tabular}{lccc}
\hline \hline$f(R)-$ Kerr & $\mathrm{R}_{0}=0$ & $\mathrm{R}_{0}=-10^{-3}$ & $\mathrm{R}_{0}=-1.2 \times 10^{-3}$ \\
\hline$r_{\text {isco }} / r_{\mathrm{g}}$ & 1.4545 & 1.4523 & 1.4518 \\
$r_{\text {Tmax }} / r_{\mathrm{g}}$ & 3.79 & 3.79 & 3.79 \\
$T_{\max }$ & $0.539 \mathrm{keV}$ & $0.54119 \mathrm{keV}$ & $0.54148 \mathrm{keV}$ \\
$E_{\max }$ & $1659.4 \mathrm{eV}$ & $1659.4 \mathrm{eV}$ & $1659.4 \mathrm{eV}$ \\
$L\left(E_{\max }\right)$ & $2.26 \times 10^{37} \mathrm{erg} \mathrm{s}^{-1}$ & $2.38 \times 10^{37} \mathrm{erg} \mathrm{s}^{-1}$ & $2.41 \times 10^{37} \mathrm{erg} \mathrm{s}^{-1}$ \\
\hline$f(R)-$ Kerr & $\mathrm{R}_{0}=-10^{-2}$ & $\mathrm{R}_{0}=-10^{-1}$ & $\mathrm{R}_{0}=-1.25 \times 10^{-1}$ \\
\hline$r_{\text {isco }} / r_{\mathrm{g}}$ & 1.4325 & 1.2017 & 1.0419 \\
$r_{\operatorname{Tmax}} / r_{\mathrm{g}}$ & 3.85 & 3.85 & 3.78 \\
$T_{\max }$ & $0.553 \mathrm{keV}$ & $0.663 \mathrm{keV}$ & $0.652 \mathrm{keV}$ \\
$E_{\max }$ & $1833.52 \mathrm{eV}$ & $2025.9 \mathrm{eV}$ & $2025.9 \mathrm{eV}$ \\
$L\left(E_{\max }\right)$ & $2.94 \times 10^{37} \mathrm{erg} \mathrm{s}^{-1}$ & $4.23 \times 10^{37} \mathrm{erg} \mathrm{s}^{-1}$ & $4.60 \times 10^{37} \mathrm{erg} \mathrm{s}^{-1}$ \\
\hline
\end{tabular}

Table 9. Values of the location of the last stable circular orbit, location in the radial coordinate of the maximum temperature, maximum temperature and luminosity, and the energy of the peak of the emission for an accretion disk around a $f(R)$-Kerr black hole with $\mathrm{R}_{0}>0$ and $\mathrm{a}=0.99$.

\begin{tabular}{lccc}
\hline \hline$f(R)$-Kerr & $\mathrm{R}_{0}=0$ & $\mathrm{R}_{0}=10^{-4}$ & $\mathrm{R}_{0}=6.67 \times 10^{-4}$ \\
\hline$r_{\text {isco }} / r_{\mathrm{g}}$ & 1.4545 & 1.4547 & 1.4559 \\
$r_{\text {Tmax }} / r_{\mathrm{g}}$ & 3.79 & 3.79 & 3.79 \\
$T_{\max }$ & $0.53942 \mathrm{keV}$ & $0.53927 \mathrm{keV}$ & $0.53843 \mathrm{keV}$ \\
$E_{\max }$ & $1659.4 \mathrm{eV}$ & $1659.4 \mathrm{eV}$ & $1659.4 \mathrm{eV}$ \\
$L\left(E_{\max }\right)$ & $2.26 \times 10^{37} \mathrm{erg} \mathrm{s}^{-1}$ & $2.25 \times 10^{37} \mathrm{erg} \mathrm{s}^{-1}$ & $2.09 \times 10^{37} \mathrm{erg} \mathrm{s}^{-1}$ \\
\hline
\end{tabular}

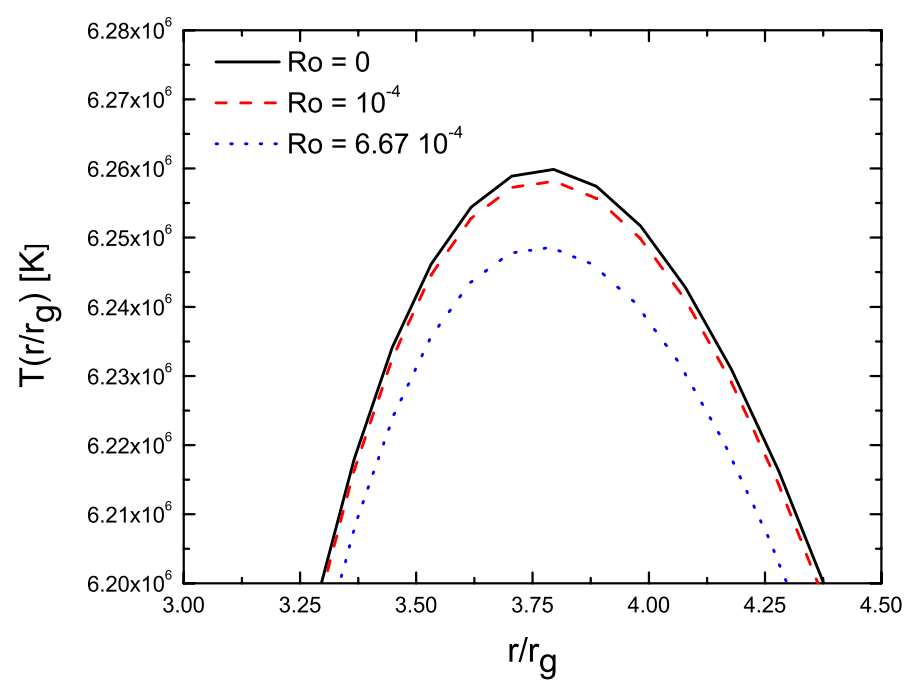

Fig. 18. Temperature as a function of the radial coordinate for some values of $\mathrm{R}_{0}>0$, for a $f(R)$-Kerr black hole of angular momentum $\mathrm{a}=0.99$.

of the peak of the emission, and in Figs. 18 and 19, the temperature and luminosity distributions respectively. As in $f(R)$ Schwarzschild black holes with positive Ricci scalar, these differences are minor.

We proceed now to examine some specific forms of the function $f$, and the constraints imposed on them by the previous analysis.

\section{Limits on specific prescriptions for $f(R)$}

As discussed in Sects. 3.1 .2 and 3.2.2, the existence of Page-Thorne disks around $f(R)$ black holes imposes the following limits on $R_{0}$ :

- $f(R)$-Schwarzschild space-time:

$$
\mathrm{R}_{0} \in\left(-\infty ; 10^{-6}\right]
$$

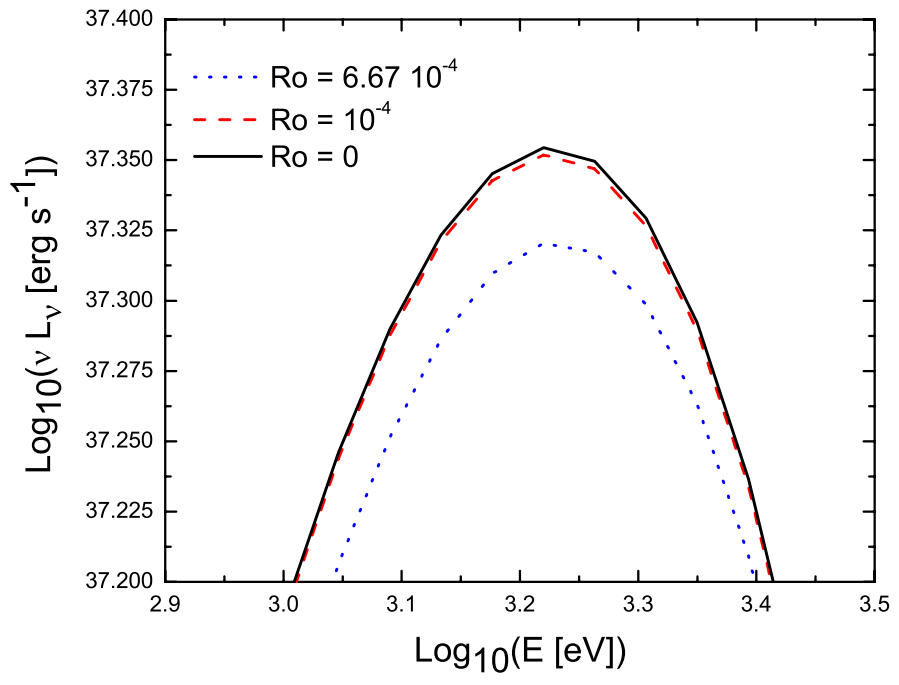

Fig. 19. Luminosity as a function of the energy for some values of $R_{0}>$ 0 , for a $f(R)$-Kerr black hole of angular momentum $\mathrm{a}=0.99$.

- $f(R)$-Kerr space-time:

$$
\mathrm{R}_{0} \in\left[-1.2 \times 10^{-3} ; 6.67 \times 10^{-4}\right]
$$

As we shall see in Sect. 6, contemporary observations of Cygnus X-1 rule out accretion disks around $f(R)$-Schwarzschild black holes, since the maximum temperature obtained in such models is lower than the inferred through observations (Gou et al. 2011). Hence, we will only consider the values of $R_{0}$ given by expression (83). We shall show in this section how these values lead to limits on the parameters of two examples of $f(R)$ theories via Eq. (7). We shall also impose the following viability conditions, to be satisfied by any $f(R)$ (Cembranos et al. 2011):

$-1<f^{\prime}\left(R_{0}\right)<0$,

$f^{\prime \prime}\left(R_{0}\right)>0$. 


\section{1. $f(R)=\alpha R^{\beta}$}

The parameters $\alpha, \beta$ and the Ricci scalar are related by Eq. (7) as follows:

$R_{0}=\left[\frac{1}{\alpha(\beta-2)}\right]^{\frac{1}{\beta-1}}$.

Introducing the adimensional parameter $\alpha^{\prime}=R_{g}^{\beta-1} \alpha$ with $R_{g} \equiv$ $r_{g}^{-2}$, this equation can be written as

$\mathbf{R}_{0}=\left[\frac{1}{\alpha^{\prime}(\beta-2)}\right]^{\frac{1}{\beta-1}}$.

Notice the condition $\beta>0$ to ensure the GR limit for small values of the Ricci scalar $R$. Let us consider first the case of a positive Ricci scalar, which leads to:

\section{Case I}

$\alpha^{\prime}>0 \wedge \beta>2$

or

$\alpha^{\prime}<0 \wedge \beta<2$,

\section{Case II}

$\alpha^{\prime}<0 \wedge \beta>2$,

or

$\alpha^{\prime}>0 \wedge \beta<2$,

and $1 /(\beta-1)$ an even number, that is:

$\beta=1+\frac{1}{2 n}$,

with $n \in \mathbb{Z}$. By isolating $\alpha^{\prime}$ from Eq. (87), we obtain the function:

$\alpha^{\prime}(\beta)=\frac{1}{\mathrm{R}_{0}^{\beta-1}}\left(\frac{1}{\beta-2}\right)$.

We show in Fig. 20 the plot of $\alpha^{\prime}$ as a function of $\beta$ (with $\beta>0$ ) for fixed values of the Ricci scalar. We see that for $\beta \in(0,2)$, $\alpha^{\prime} \in(-\infty, 0)$. For $\beta=2$, Eq. (93) is not defined, while for $\beta>2$, $\alpha^{\prime}$ takes large positive values. Since $\alpha$ needs to be small in order to recover GR for small values of the Ricci scalar, the case $\alpha^{\prime}>$ $0, \beta>2$ is discarded. Hence, we obtain the following restrictions on the parameters:

$$
\alpha^{\prime} \in(-\infty ; 0) \wedge \beta \in(0 ; 2) \wedge \mathrm{R}_{0} \in\left(0 ; 6.67 \times 10^{-4}\right] .
$$

For negative values of the Ricci scalar, from Eq. (93) we require that:

$1-\beta=2 m, \Rightarrow \beta_{\text {odd }}=1-2 m$,

or:

$1-\beta=2 m+1, \Rightarrow \beta_{\text {even }}=-2 m$,

where $m \in \mathbb{Z}_{0}^{-}$, so that $\beta>0$. If $m=0, \beta=1$ and $\alpha^{\prime}=\alpha=-1$. These values of the parameters lead to $f(R)=-R$, which does not reduce to GR. For $\beta=2$, Eq. (93) is not defined. If $\beta \geq 3$ and is an odd number, $\alpha^{\prime}$ takes positive large values, while if $\beta \geq 4$ and is an even number $\alpha^{\prime}$ is large and negative. Since $\alpha^{\prime}$ has

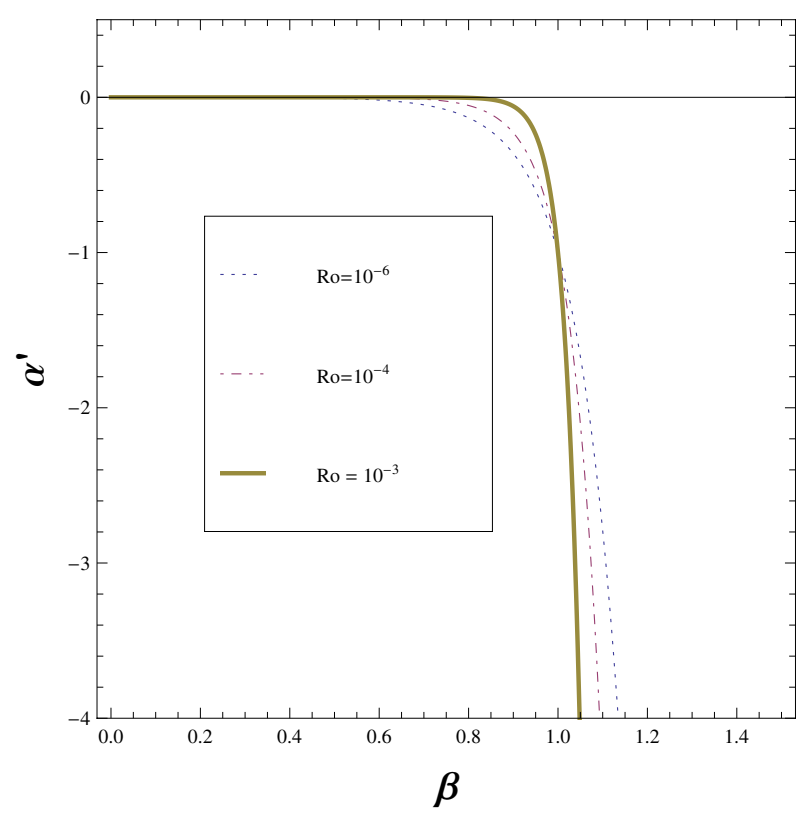

Fig. 20. $\alpha^{\prime}$ as a function of $\beta$ for different values of $\mathbf{R}_{0}$.

to be small to recover GR for small values of the Ricci scalar, we conclude that negative values of $R_{0}$ are not allowed in this theory.

We now restrict the values of $\alpha$ and $\beta$ according to Eqs. (84) and (85). The first and second derivative for the given $f$ function are:

$f^{\prime}(R)=\alpha \beta R^{\beta-1}$,

$f^{\prime \prime}(R)=\alpha \beta(\beta-1) R^{\beta-2}$.

The restrictions over $\alpha$ and $\beta$ that satisfy Eq. (85) are:

$\alpha>0 \wedge \beta>1$,

or

$\alpha<0 \wedge \beta \in(0,1)$.

The condition given by Eq. (98) is discarded because it does not satisfy Eq. (93). The viability condition expressed by Eq. (84) takes the form:

$-1<\alpha \beta R_{0}{ }^{\beta-1}<0$.

We can constrain the values of $\alpha$ using the latter inequality as follows:

$0<\beta<1$.

By multiplying by $R_{0}^{\beta-1} \alpha$ the latter restrictions yields:

$0>\alpha \beta R_{0}{ }^{\beta-2}>\alpha R_{0}{ }^{\beta-1}$.

In order to satisfy Eq. (100):

$\alpha R_{0}^{\beta-1}>-1 \Rightarrow \alpha>\frac{-1}{R_{0}{ }^{\beta-1}}$.

If $\beta=0, \alpha>-R_{0}$, and for $\beta=1, \alpha>-1$. The set of values for $\alpha$ is $\alpha \in\left(-R_{0}, 0\right)$.

We conclude that the values of $\alpha$ and $\beta$ that are permitted by our model as well as by the two viability conditions are:

$$
\alpha \in\left(-R_{0} ; 0\right) \wedge \beta \in(0 ; 1) \wedge \mathrm{R}_{0} \in\left(0 ; 6.67 \times 10^{-4}\right] .
$$




\section{2. $f(R)=R \epsilon \ln \frac{R}{\alpha}$}

In this case, the parameters $\epsilon$ and $\alpha$, and the Ricci scalar are related by Eq. (7) in the simple form:

$\alpha=R_{0} \exp \left(\frac{1}{\epsilon}-1\right)$.

Dividing by $R_{g}$, we obtain $\alpha^{\prime}=\mathrm{R}_{0} \exp \left(\frac{1}{\epsilon}-1\right)$. For $\mathrm{R}_{0}>0$, the function $\alpha^{\prime}(\epsilon)$ is always positive, while it is negative for all $\epsilon$ if $\mathrm{R}_{0}<0$. The constrains over $\epsilon$ and $\alpha$ that follow from Eq. (7) are:

$$
\begin{aligned}
& -\mathrm{R}_{0} \in\left(0 ; 6.67 \times 10^{-4}\right] \\
& \quad \epsilon \in(-\infty ; 0) \wedge \alpha^{\prime} \in \\
& \text { or } \\
& \quad \epsilon \in(0 ; \infty) \wedge \alpha^{\prime} \in( \\
& -\mathrm{R}_{0} \in\left[-1.2 \times 10^{-3} ; 0\right) \\
& \quad \epsilon \in(-\infty ; 0) \wedge \alpha^{\prime} \in \\
& \text { or } \\
& \quad \epsilon \in(0 ; \infty) \wedge \alpha^{\prime} \in( \\
& \text { The first and second deriv } \\
& f^{\prime}(R)=\epsilon\left(1+\ln \frac{R}{\alpha}\right), \\
& f^{\prime \prime}(R)=\frac{\epsilon}{R} .
\end{aligned}
$$$$
\epsilon \in(-\infty ; 0) \wedge \alpha^{\prime} \in\left(0 ; e^{-1} \mathrm{R}_{0}\right),
$$$$
\epsilon \in(0 ; \infty) \wedge \alpha^{\prime} \in\left(e^{-1} \mathrm{R}_{0} ; \infty\right),
$$$$
\epsilon \in(-\infty ; 0) \wedge \alpha^{\prime} \in\left(-e^{-1}\left|\mathrm{R}_{0}\right| ; 0\right),
$$

$$
\epsilon \in(0 ; \infty) \wedge \alpha^{\prime} \in\left(-\infty ;-e^{-1}\left|\mathrm{R}_{0}\right|\right) .
$$

The first and second derivative of the function $f$ take the form:

The condition $f^{\prime \prime}\left(R_{0}\right)>0$ is satisfied if $\epsilon>0 \wedge R_{0}>0$, or $\epsilon<0 \wedge R_{0}<0$. Equation (84) in adimensional form is:

$-1<\epsilon\left(1+\ln \frac{\mathrm{R}_{0}}{\alpha^{\prime}}\right)<0$.

This equation, together with (104), yields:

$$
\begin{aligned}
& \text { - For } \epsilon>0 \text { and } \mathrm{R}_{0}>0, \alpha^{\prime} \in\left(e \mathrm{R}_{0} ; \mathrm{R}_{0} \exp \left\{\left(\frac{1}{\epsilon}+1\right)\right\}\right) \text {. } \\
& \text { - For } \epsilon<0 \text { and } \mathrm{R}_{0}<0, \\
& \alpha^{\prime} \in\left(-e\left|\mathrm{R}_{0}\right| ;-\left|\mathrm{R}_{0}\right| \exp \left\{1-\frac{1}{|\epsilon|}\right\}\right) .
\end{aligned}
$$

Summarizing all the constraints, we have that:

$$
\begin{aligned}
& \mathrm{R}_{0} \in\left(0 ; 6.67 \times 10^{-4}\right], \quad \epsilon>0, \quad \alpha^{\prime} \in\left(e^{-1} \mathrm{R}_{0} ; \infty\right), \\
& \alpha^{\prime} \in\left(e \mathrm{R}_{0} ; \mathrm{R}_{0} \exp \left\{\left(\frac{1}{\epsilon}+1\right)\right\}\right),
\end{aligned}
$$

and

$$
\begin{aligned}
& \mathrm{R}_{0} \in\left[-1.2 \times 10^{-3} ; 0\right), \quad \epsilon<0, \quad \alpha^{\prime} \in\left(-e^{-1}\left|\mathrm{R}_{0}\right| ; 0\right) \\
& \alpha^{\prime} \in\left(-e\left|\mathrm{R}_{0}\right| ;-\left|\mathrm{R}_{0}\right| \exp \left\{1-\frac{1}{|\epsilon|}\right\}\right)
\end{aligned}
$$

The first group of constraints is fulfilled for $\epsilon>0$, while in the second group for only $\epsilon \in(-1 / 2,0)$. We conclude that for the $f(R)$ under scrutiny, the values

$\mathrm{R}_{0} \in\left(0 ; 6.67 \times 10^{-4}\right], \quad \epsilon>0, \quad \alpha^{\prime} \in\left(e \mathrm{R}_{0} ; \infty\right)$,

and,

$\mathrm{R}_{0} \in\left[-1.2 \times 10^{-3} ; 0\right), \quad \epsilon \in(-1 / 2,0), \quad \alpha^{\prime} \in\left(-e^{-1}\left|\mathrm{R}_{0}\right| ; 0\right)$

are allowed.

\section{Discussion}

The results presented in Sect. 4 can be compared with current observational data to derive some constraints on a given $f(R)$ theory. In order to illustrate this assertion we shall consider Cygnus $\mathrm{X}-1$, which is the most intensively studied black hole binary system in the Galaxy. A series of recent high-quality papers (Reid et al. 2011; Orosz et al. 2011; Gou et al. 2011) have provided an unprecedented set of accurate measurements of the distance, the black hole mass, spin parameter a, and the orbital inclination of this source. This opens the possibility to constrain modified theories of gravity with rather local precision observations of astrophysical objects in the Galaxy.

Cygnus X-1 was discovered at X-rays by Bowyer et al. (1965). Early dynamical studies of the compact object suggested the presence of an accreting black hole (e.g. Bolton 1972). The distance to Cygnus X-1 is currently estimated to be $1.86_{-0.11}^{+0.12} \mathrm{kpc}$ (Reid et al. 2011). This value was determined via trigonometric parallax using the Very Long Baseline Array (VLBA). At this distance, the mass of the black hole is (Orosz et al. 2011) $M=14.8 M_{\odot}$. This is the value adopted in all calculations presented in the previous sections.

The source has been observed in both a low-hard state, dominated by the emission of a hot corona (e.g., Dove et al. 1997; Gierlinski et al. 1997; Poutanen 1998), and a high-soft state, dominated by the accretion disk, which in this state goes all the way down to the last stable orbit. In the low-hard state, where the source spends most of the time, a steady, non-thermal jet is observed (Stirling et al. 2001). The jet is absent in the thermal state. Therefore, in this latter state a clearer X-ray spectrum can be obtained.

The accretion rate and the spin parameter of the hole are $\sim 0.472 \times 10^{19} \mathrm{~g} \mathrm{~s}^{-1}$ and 0.99 , respectively, according to estimates from a Kerr plus black-body disk model (Gou et al. 2011). These GR models yield a spectral energy distribution with a maximum at $E_{\max } \sim 1.6 \mathrm{keV}$. On the contrary, $f(R)$-models with negative curvature correspond to a low maximum temperature, lower even than what is expected for the (unrealistic) case of a Schwarzschild black hole. Therefore, we can presume that a fit of $f(R)$-Kerr models to the data would also prefer high values of maximum temperature, i.e., ones with non-negative curvature. Models with accretion rates and spin close to those obtained by Gou et al. (2011) and small positive curvature seem viable, something that is consistent with an asymptotic behaviour corresponding to a de Sitter space-time endowed with a small and positive value of the cosmological constant.

Deep X-ray studies with Chandra satellite might impose more restrictive limits, especially if independent constraints onto the accretion rate become available.

\section{Conclusions}

We have studied stable circular orbits and relativistic accretion disks around Schwarzschild and Kerr black holes in $f(R)$ gravity with constant Ricci curvature in the strong regime. We have found that stable disks can be formed only for curvatures in the ranges of $\left(-\infty, 10^{-6}\right]$ and $\left[-1.2 \times 10^{-3}, 6.67 \times 10^{-4}\right]$ in the cases of Schwarzschild and Kerr black holes, respectively. Current observations of Cygnus X-1 in the soft state rule out curvature values below $-1.2 \times 10^{-3}$. Additional constrains can be imposed on specific prescriptions of $f(R)$ gravity. In particular, logaritmicgravity prescriptions are strongly constrained by observational data. Future high-precision determination of the parameters of 
other black hole candidates can be used to impose more restrictive limits to extended theories of gravity.

Acknowledgements. We are grateful to an anonymous referee for useful suggestions. BH astrophysics with G.E. Romero is supported by grant PIP 2010/0078 (CONICET). Additional funds comes from Ministerio de Educación y Ciencia (Spain) trhough grant AYA 2010-21782-C03-01. S.E.P.B. acknowledges support from UERJ, FAPERJ, and ICRANet-Pescara. We thank Gabriela Vila and Florencia Vieyro for comments on accretion disks.

\section{References}

Abdelwahab, M., Goswami, R., \& Dunsby, P. 2012, Phys. Rev. D, 85, 083511 Arbuzova, E. V., Dolgov, A. D., \& Reverberi, L. 2012, JCAP, 02, 04

Biswas, T., Cembranos, J. A. R., \& Kapusta J. I. 2010, Phys. Rev. Lett., 104, 021601

Biswas, T., Cembranos, J. A. R., \& Kapusta, J. I. 2010, JHEP, 1010, 048

Bolton, C. T. 1972, Nat. Phys. Sci., 240, 124

Bowyer, S., Byram, E. T., Chubb, T. A., \& Friedman H. 1965, Science, 147, 394

Capozziello, S. 2002, Int. J. Mod. Phys. D, 11, 483

Capozziello S., \& Faraoni V. 2010, Beyond Einstein Gravity. A survey of Gravitational Theories for Cosmology and Astrophysics (DordrechtHeidelberg-London-New York: Springer)

Carter, B. 1973, in Les Astres Occlus eds. C. DeWitt, \& B. DeWitt (New York, Gordon \& Breach)

Cembranos, J. A. R., de la Cruz-Dombriz, A., \& Jimeno Romero, P. 2011 [arXiv: 1109.4519v1]

Cooney, A., DeDeo, S., \& Psaltis D. 2010, Phys. Rev. D, 82, 064033

De Felice, A., \& Tsujikawa, S. 2010, Living Rev. Relat., 133

de la Cruz-Dombriz, A., Dobado, A., \& Maroto, A. L. 2009, Phys. Rev. D, 80, 124011; Erratum-ibid, 2011, 83, 029903

Dove, J. B., Wilms, J., Maisack, M., \& Begelman, M. 1997, ApJ, 487, 759

Gierlinski, M., Zdziarski, A. A., Done, C., et al. 1997, MNRAS, 228, 958

Gou, L., McClintock, J. E., Reid, Mark J., et al. 2011, ApJ, 742, 85

Harko, T., Kovács, Z., \& Lobo, F. N. N. 2009, Phys. Rev. D, 79, 064001

Hendi, S. H., \& Momeni, D. 2011, Eur. Phys. J. C, 71, 1823
Hendi, S. H., Panah, B. E., \& Mousavi, S. M. 2012, Gen. Rel. Grav., 44, 835 Hobson, M. P., Estathoiou, G. P., \& Lasenby, A. N. 2006, General Relativity. An Introduction for Physicists (Cambridge: Cambridge, University Press)

Kerr, R. P. 1963, Phys. Rev. Lett., 11, 237

Li, M., Li, X., \& Wang, S. 2011, Commun. Theor. Phys., 56, 525

Magnano, G., Ferraris, M., \& Francaviglia, M. 1987, Gen. Rel. Gravit., 19, 465 Mazharimousavi, H. S., Halilsay, M., \& Tahamtan, T. 2012, Eur. Phys. J. C, 72, 1851

Moon, T., Myung, Y. S., \& Son, E. J. 2011a, Gen. Rel. Gravit., 43, 3079

Moon, T., Myung, Y. S., \& Son, E. J. 2011b, Eur. Phys. J., C71, 1777

Motohashi, H., \& Nishizawa, A. 2012, Phys. Rev. D, 86, 083514

Myung, Y. S. 2011, Phys. Rev. D, 84, 024048

Myung, Y. S., Moon, T., \& Son, E. J. 2011, Phys. Rev. D, 83, 124009

Novikov, I. D., \& Thorne, K. S. 1973, in Black Holes, eds. C. DeWitt, \& B. DeWitt (New York: Gordon \& Breach)

Orosz, J. A., McClintock, J. E., Aufdenberg, J. P., et al. 2011, ApJ, 742, 84

Page, D. N., \& Thorne, K. S. 1974, ApJ, 191, 499

Perez Bergliaffa, S. E., \& Yves De Oliveira, E. 2011, Phys. Rev. D, 84, 084006

Poutanen, J. 1998, Theory of Black Hole Accretion Disks, eds. M. A. Abramowicz, Gunnlaugur Bjornsson, and James E. Pringle. (Cambridge: Cambridge University Press)

Psaltis, D., Perrodin, D., Dienes, K. R., \& Mocioiu, I. 2008a, Phys. Rev. Lett., 100,091101

Psaltis, D., Perrodin, D., Dienes, K. R., \& Mocioiu, I. 2008b, Phys. Rev. Lett., 100,119902

Pun, C. S., Zovács, Z., \& Harko, T. 2008, Phys. Rev. D, 78, 024043

Reid, M. J., McClintock, J. E., Narayan, R., et al. 2011, ApJ, 742, 83

Rezzolla, L., Zanotti, O., \& Font, J. A. 2003, A\&A, 412, 603

Schwarzschild, K. 1916, Sitzungsberichte der Königlich-Preussischen Akademie der Wissenschaften, 3, 189

Shakura, N. I., \& Sunyaev, R. A. 1973, A\&A, 24, 377

Sotiriou, T. P., \& Faraoni, V. 2010, Rev. Mod. Phys., 82, 451

Starobinsky, A. A. 1980, Phys. Lett. B, 91, 99

Stirling, A. M., Spencer, R. E., de la Force, C. J., et al. 2001, MNRAS, 327, 1273

Stuchlík, Z., \& Hledík, S. 1999, Phys. Rev. D, 60, 044006

Stuchlík, Z., \& Slaný, P. 2004, Phys. Rev. D, 69, 064001

Slaný, P., \& Stuchlík, Z. 2005, Class. Quant. Grav., 22, 3623

Weinberg, S. 1989, Rev. Mod. Phys., 61, 1

Will, C. M. 2006, Living Rev. Relat., 9, 3 